\title{
Review
}

\section{Recent progress in photodetectors based on low-dimensional nanomaterials}

https://doi.org/10.1515/ntrev-2018-0084

Received May 31, 2018; accepted August 13, 2018; previously published online September 12, 2018

\begin{abstract}
Photodetectors (PDs) have great potential in applications of imaging, telecommunication, and biological sensing. In this article, state-of-the-art achievements on typical low-dimensional nanostructured PDs and hybrid PDs are reviewed. In the 2D nanostructured PDs part, 2D transition metal dichalcogenides have a natural gap, which promise high sensitivity of photodetection. Graphene and black phosphorus can also stand for 2D nanostructured PDs due to their broadband absorption and tunable direct bandgap, respectively. In the 1D nanostructured PDs part, owing to its high photoconductive characteristic, $\mathrm{ZnO}$ nanowire film is a promising material for ultraviolet PDs. Carbon nanotubes show potential in infrared (IR) detection due to its unique physical properties. In the OD nanostructured PDs part, lead sulfide has a small bandgap and large Bohr exciton radius, which collectively give it a wide spectral tunability in the IR. In the hybrid PDs part, electrical and chemical doping is applied to combine different nanomaterials to realize PDs with high performance. In each part, the present situation and major challenges are overviewed. Then, the evolutions of the methods to overcome these challenges and the tremendous research breakthroughs are demonstrated. At last, future directions that could improve the performance of PDs are discussed.
\end{abstract}

Keywords: nanomaterials; photodetectors; typical low dimensional.

\footnotetext{
*Corresponding author: Ke Xu, School of Information and Control Engineering, Shenyang Jianzhu University, Shenyang, China, e-mail: xksky1234@163.com

Zhenhui Li: School of Information and Control Engineering, Shenyang Jianzhu University, Shenyang, China

Fanan Wei: School of Mechanical Engineering and Automation, Fuzhou University, Fuzhou, China
}

\section{Introduction}

During the past several decades, the booming photoelectronic industry has stretched throughout every facet of our lives. As one of the most critical optoelectronic devices, photodetectors (PDs), which can transform light into electrical signals precisely, have been stimulated into extensive research. Up to now, the accurate detection of light from the ultraviolet (UV; $10-400 \mathrm{~nm}$ ) to the visible $(400-760 \mathrm{~nm})$ to the infrared (IR; $760-1 \mathrm{~mm}$ ) to the terahertz $(0.1-10 \mathrm{THz})$ region is critical for extensive and significant applications in military and civil use, such as night vision, imaging, sensing, and even information communication systems. Under the impetus of Moore's law, the feature size of highly integrated electronic devices has shrunk toward several nanometers. For example, Intel has achieved high volume production of $10 \mathrm{~nm}$ chips, and IBM Research Alliance produced the industry's $7 \mathrm{~nm}$ node test chips. Therefore, a major current focus in developing PDs is how to scale them down to the nanoscale for technological applications to realize a highly integrated photodetection system with high performance [1-4].

It has widely been shared that one way to improve the performance of PDs is to explore new low-dimensional nanomaterials [5]. The other way is to use hybridized structures. With one spatial dimension confined within the nanoscale, 2D materials, especially in terms of 2D layered materials and solution-processed nanofilms, exhibit many unique favorable properties for photodetection. Compared to the growth of conventional semiconductor films via universal epitaxial techniques, 2D materials are usually fabricated by simpler methods [6-8]. In addition, they have exceptional potential in electronic and optoelectronic applications [9]. For example, graphene exhibits extremely high charge-carrier mobility, optical transparency, and broadband absorption $[10,11]$. In contrast to 2D nanostructured materials, having 1D limited within the nanoscale, 1D nanostructure materials possess an additional spatial dimension in total 2D confined within the nanoscale. As a result, they are ideal systems for exploring a large 
number of novel phenomena at the nanoscale as well as investigating the size and dimensionality dependence of properties for potential applications. In addition, its large surface-to-volume ratio and Debye length comparable to their sizes are also attractive. OD nanostructured materials usually refer to materials whose excitons are confined in all three spatial dimensions $(<100 \mathrm{~nm})$. The nanoscale confinement of OD materials makes it possible to tune its energy band by its size and shape. As a result of this 3D tunability of OD materials, they usually exhibit several unique electronic and optical properties, especially for use of PDs $[8,9]$. Hybrid method can help improve the performance of present low-dimensional PDs; for instance, to yield an ultrahigh responsivity of PDs, a special hybrid configuration consisting of 2D quantum dot (QD) materials was demonstrated [12]. The aim of this review paper is to present state-of-the-art PDs based on low-dimensional nanomaterials, including graphene, transition metal dichalcogenides (TMDCs), black phosphorus (BP), ZnO nanowire film, carbon nanotube (CNT), and lead sulfide $(\mathrm{PbS})$. Moreover, the hybrid method incorporated in this field is demonstrated in detail as well. Here, the performance of PDs is measured by " $5 \mathrm{~S}$ " requirements: sensitivity, signal-to-noise ratio (SNR), spectral selectivity, speed, and stability. In the last part, the possible challenges and opportunities in the future development of these PDs are proposed.

\section{2D nanostructured PDs}

\subsection{Graphene}

Recently, graphene has emerged as an attractive material in PDs due to its high carrier mobility, broadband absorption, and mechanical flexibility. Due to graphene's typical plasmonic nanostructures, photocurrent is no longer limited to photon energies greater than the bandgap of the semiconductor [9]. Moreover, it can be integrated into virtually any waveguide material, including $\mathrm{Si}, \mathrm{SiN}$, or AlN. Early data interpreted in the study have revealed an ultrafast photoresponse in graphene with an intrinsic bandwidth of $260 \mathrm{GHz}$, showing the promise for high-speed photodetection applications. Since then, several graphene-based PDs on Si waveguides have been realized [12].

Schuler et al. [13] presented an ultrafast graphenebased PD on an Si slot waveguide, where the Si strips serve as local back gate electrodes to create a p-n junction for efficient photodetection. A responsivity of $35 \mathrm{~mA} / \mathrm{W}$ at zero-bias conditions was achieved, and while under a moderate drain-source bias of $300 \mathrm{mV}$, the responsivity increased to $76 \mathrm{~mA} / \mathrm{W}$. This PD has shown a record high $3 \mathrm{~dB}$ bandwidth of $65 \mathrm{GHz}$. To further improve the responsivity in terms of $\mathrm{V} / \mathrm{W}$, the electrical gating could be extended closer to the contacts, for example, using thin $\mathrm{Si}$ slabs to both sides of the waveguide.

However, the realization of broadband PDs has been hindered by graphene's low absorption in the visible and near-IR (NIR) regions. Kang et al. [14] demonstrated a stretchable PD based exclusively on crumpled graphene, which solved this barrier perfectly. As shown in Figure 1, the crumpled graphene, which has been intentionally deformed to have a continuously undulating 3D surface, induced an areal density increase to yield higher optical absorption per unit area, thereby improving photoresponsivity. As a result, this textured graphene PD exhibited a $370 \%$ enhancement in photoresponsivity compared to a flat graphene PD. More importantly, the stretching capability could reach up to $200 \%$ of its original length and no limit on detection wavelength. This surface engineering approach can be used to enhance photoabsorption and develop a highly responsive PD based on a single photoactive material of other emerging 2D materials such as TMDCs, including $\mathrm{MoS}_{2}, \mathrm{WS}_{2}$, and $\mathrm{WSe}_{2}$ monolayers.

Fang et al. [15] demonstrated a broadband graphene PD exhibiting enhancement factors of 8 to 13 over the visible spectrum by reforming the source and drain contacts into a fractal metasurface and a ring encircling it. The metasurface was realized through a fractal tree to mimic the snowflake geometry, as shown in Figure 2. The application of this fractal metasurface was not bound to graphene PDs but could also be integrated with photovoltaic/photothermoelectric effect PDs made of other photodetection materials. The great flexibility of our fractal metasurface design enabled broadband enhancement at other portions of the electromagnetic spectrum and for various spot sizes. Additionally, the photoresponse enhancement was insensitive to the incident light polarization, a quintessential feature of a practical photodetecting/photoharvesting system. These attributes, combined with dynamic tunability through source-drain bias, make this fractal metasurface an advancement toward the incorporation of graphene into modern photodetecting and photoharvesting applications.

\subsection{TMDCs}

Great improvement in the responsivity has been realized for PDs based on graphene; however, there is an intractable 
A
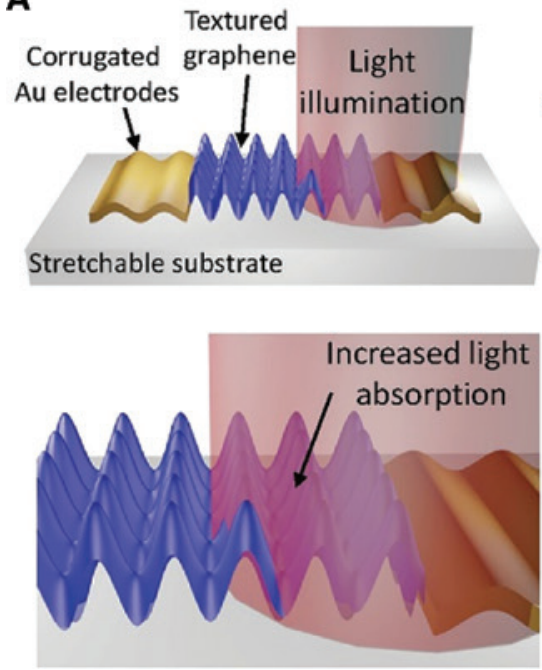
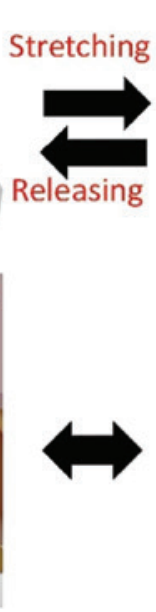

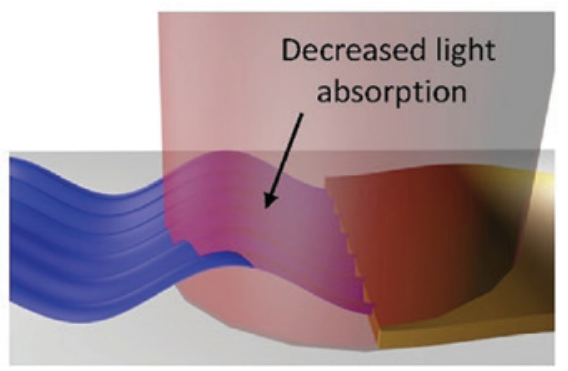

B

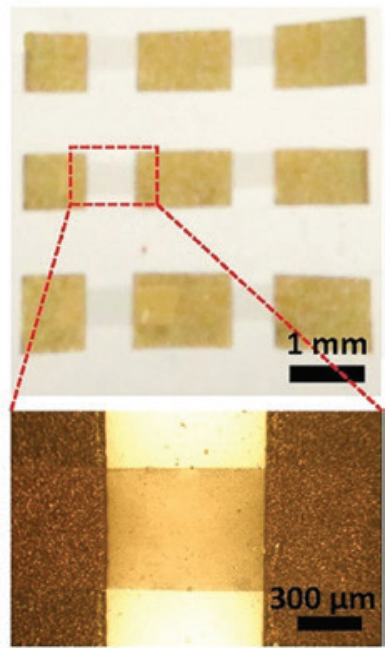

Figure 1: Mechanism of enhanced and strain-tunable photoresponsivity and photographs of textured graphene PD arrays. (A) Schematic illustrations of the physical mechanism enabling enhanced and strain-tunable photoresponsivity. Bottom schematic illustrations show close-up at the interface and illustrate the modulation of crumple areal density, height, and pitch in the illuminated region with an applied strain. (B) Photograph of a stretchable PD array (3*2 array). Bottom image shows an optical microscope image of the textured graphene PD. Reproduced with permission from Ref. [14].
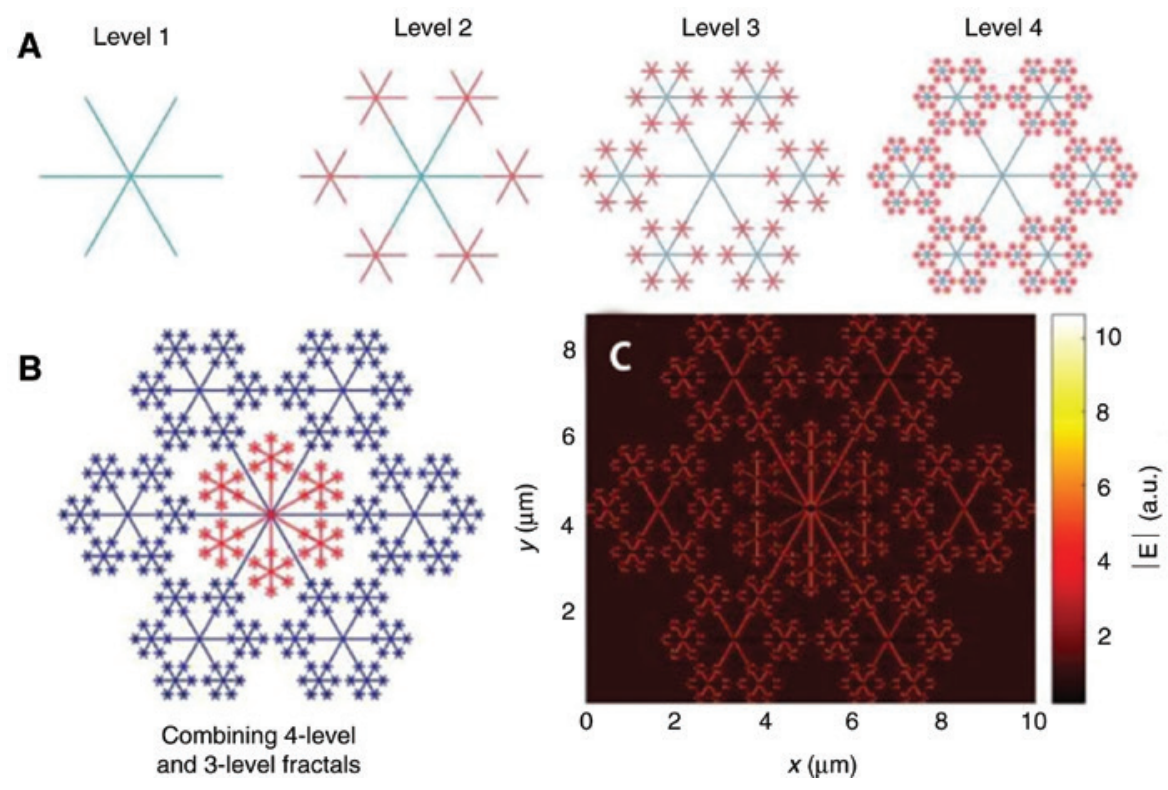

Figure 2: Construction of the branching generated by recursive iterations. (A) Construction of the fractal design with "snowflake" geometry from levels 1-4. (B) Total structure comprising four-level (blue) and three-level (red) fractals used in the study. (C) Finite-difference timedomain simulated in-plane electric field (of the incident electromagnetic wave) distribution just underneath the Au fractal metasurface on a glass substrate under the excitation wavelength of $530 \mathrm{~nm}$. The electric field is linearly polarized along the $y$-direction. Reproduced with permission from Ref. [15].

issue for graphene-based devices: the high dark current arising from its gapless nature, which has a strong impact on the sensitivity of photodetection [16, 17]. Alternatively, another kind of layered material, TMDCs with a natural gap, has captured much more attention in recent years. Up to now, TMDCs, including $\mathrm{MoS}_{2}, \mathrm{WS}_{2}$, WSe $\mathrm{2}_{2}$ InSe, GaS,
GaTe, GaSe, and $\mathrm{In}_{2} \mathrm{Se}_{3}[18]$, have been explored to develop plenty of new PDs with favorable photoelectronic properties. To illustrate this kind of PD vividly, $\mathrm{MoS}_{2}$-based PDs are taken as an example.

As a typical TMDC material, $\mathrm{MoS}_{2}$ is composed of a vertical stack in which the weakly interacting layers are 
held together by van der Waals forces. It is noteworthy that bulk-form $\mathrm{MoS}_{2}$ is a semiconductor with an indirect bandgap of $1.2 \mathrm{eV}$, whereas single-layer $\mathrm{MoS}_{2}$ is another kind of semiconductor with a direct bandgap of $1.8 \mathrm{eV}$ [19]. This unique property of $\mathrm{MoS}_{2}$ stems from the effect of quantum confinement on its electronic structure.

Ye et al. [20] demonstrated an advanced highly polarization-sensitive IR PD based on BP-on-WSe ${ }_{2}$ van der Waals vertical heterostructure. This PD used a vertical photogate heterostructure of BP-on-WSe ${ }_{2}$ in which $\mathrm{BP}$ serves as the photogate and $\mathrm{WSe}_{2}$ as the conductive channel. Ultrahigh visible and IR photoresponsivity at room temperature can reach up to 1000 and $0.5 \mathrm{~A} / \mathrm{W}$, respectively, and ultrasensitive visible and IR specific detectivity is obtained up to $10^{14}$ and $10^{10}$ Jones, respectively, at room temperature. Moreover, the high sensitivity to IR polarization is about $40 \mathrm{~mA} / \mathrm{W}$ with incident light polarized along the horizontal axis (defined as $0^{\circ}$ polarization). It shows that the BP photogate structure of the BP-on-WSe ${ }_{2}$ detector has higher sensitivity to polarized IR illumination photodetection and lower specific detectivity at room temperature. This detector provides a new strategy to integrate 2D layered materials to enable the corresponding PDs and may open up a new dimension for applications to IR imaging systems operating at room temperature.

Gomathi et al. [21] fabricated a novel paper-based broadband PD using hybrids of $\mathrm{ZnS}-\mathrm{MoS}_{2}$. The PD was fabricated using a simple two-step hydrothermal method. The spectral selectivity of $\mathrm{MoS}_{2}$ has been extended to UV wavelength region by combining $\mathrm{MoS}_{2}$ with $\mathrm{ZnS}$ having high sensitivity toward UV light. The fabricated PD displays high sensitivity toward visible light when compared to UV and IR. The PD exhibits a responsivity of $4.5 \mu \mathrm{A} / \mathrm{W}$ for IR, $9.4 \mu \mathrm{A} / \mathrm{W}$ for UV light, and $17.85 \mu \mathrm{A} / \mathrm{W}$ for visible light. In addition, $\mathrm{ZnS}-\mathrm{MoS}_{2}$ exhibited increased responsivity due to the reduced electron-hole recombination, which was a result of the straddling-type band alignment observed at the interface of ZnS-MoS . This is the first report on paper-based broadband PD with $\mathrm{ZnS}-\mathrm{MoS}_{2}$ hybrids as active sensing materials and it provides a promising route for the development of large-scale paper-based broadband PDs using TMDC hybrids at low cost, having diverse applications in the field of wearable electronics, environmental monitoring, and surveillance.

\section{$2.3 \mathrm{BP}$}

In addition to TMDC layered materials, BP is the latest developed layered material [22, 23]. After the groundbreaking research on BP in 2004 by Manchester scientists, this layered material opened a new branch of science and provided a vast number of unlimited applications ranging from printable electronics to prototype devices [3].

In contact to other 2D nanomaterials, $\mathrm{BP}$ has intrinsic and tunable direct bandgap from $2.0 \mathrm{eV}$ (monolayer) to $0.3 \mathrm{eV}$ (bulk), which covers the optical spectrum range from visible to NIR. In addition, BP has also shown remarkable electrical properties, including a high hole mobility up to $1000 \mathrm{~cm}^{2} \mathrm{~V}^{-1} \mathrm{~s}^{-1}$ at room temperature, an on-off ratio up to 105, and good current saturation in field-effect devices [24]. BP-based field-effect transistors (FETs) and their performances are shown in Table 1. Furthermore, it has unique in-plane anisotropic physical properties unlike most of other 2D materials. The anisotropy of any physical property refers to the change of that property in different directions. It is not new in materials but is quite exciting in new 2D material BP due to its large magnitude in anisotropy compared to other 2D materials [3]. These characteristics make $\mathrm{BP}$ as the most promising candidate for broadband photodetection.

However, the current performance status of BP PDs in terms of broadband spectrum range, high photoresponsivity, and fast response time is still unsatisfactory. Furthermore, the impurities and the dielectric environment have an important impact on the electrical and optical properties of BP devices. In this section, we attempt to give a comprehensive overview and analysis of the published results of BP PD and show some new pathways to overcome these challenges.

Table 1: BP-based FETs and their performances.

\begin{tabular}{|c|c|c|c|}
\hline $\begin{array}{l}\text { Film } \\
\text { thickness (nm) }\end{array}$ & $\begin{array}{r}\text { Channel } \\
\text { length }(\mu \mathrm{m})\end{array}$ & $\begin{array}{l}\text { Gate } \\
\text { insulator }\end{array}$ & $\begin{array}{l}\text { Hole mobility } \\
\left(\mathrm{cm}^{2} \mathrm{~V}^{-1} \mathrm{~s}^{-1}\right)\end{array}$ \\
\hline 10 & 1.6 & $\mathrm{SiO}_{2}$ & 984 \\
\hline 5 & 1 & $\mathrm{SiO}_{2}$ & 286 \\
\hline 5 & 1 & $\mathrm{SiO}_{2}$ & 205 \\
\hline 18.7 & 3 & $\mathrm{SiO}_{2}$ & 170 \\
\hline 5 & 0.69 & $\mathrm{SiO}_{2}$ & 155 \\
\hline 1.6 & 0.45 & $\mathrm{SiO}_{2}$ & 35 \\
\hline 8.5 & 0.3 & $\mathrm{HfO}_{2}$ & 400 \\
\hline 1.9 & 2 & $\begin{array}{l}\mathrm{SiO}_{2} \text { (back)/ } \\
\mathrm{Al}_{2} \mathrm{O}_{3} \text { (top) }\end{array}$ & 172 \\
\hline 15 & 2.7 & $\begin{array}{l}\text { Polyimide } \\
\text { (back) } / \mathrm{Al}_{2} \mathrm{O}_{3} \\
\text { (top) }\end{array}$ & 310 \\
\hline 5 & & & 180 \\
\hline$\sim 5$ & - & Ionic liquid & 510 \\
\hline 8 & - & $\mathrm{h}-\mathrm{BN}$ & 1350 \\
\hline 10 & - & h-BN & 400 \\
\hline
\end{tabular}

Reproduced with permission from Ref. [3]. 
Miao et al. [25] fabricated an NIR camera based on a single BP PD using the compressive sensing algorithm, which took advantage of the outstanding photoresponse of BP and overcame the difficulties of BP film growth and scalable PD array fabrication. The imaging was achieved by combining the PD with a digital micromirror device to encode and subsequently reconstruct the image based on compressive sensing algorithm. Stationary images of an NIR laser spot $(\lambda=830 \mathrm{~nm})$ with up to $64 \times 64$ pixels were captured using this single-pixel BP camera with 2000 times of measurements, which was only half of the total number of pixels. The imaging platform demonstrated in this work circumvents the grand challenges of scalable BP material growth for PD array fabrication and shows the efficacy of using the outstanding performance of BP PD for future high-speed IR camera applications.

Ren et al. [2] synthesized few-layer BP nanosheets with large size by a facile liquid exfoliation method. Photoelectrochemical (PEC) tests demonstrated that the current density of BP nanosheets can reach up to $265 \mathrm{nA} \mathrm{cm}^{-2}$ under light irradiation, whereas the dark current densities fluctuated near 1 $\mathrm{nA} \mathrm{cm}{ }^{-2}$ in $0.1 \mathrm{M} \mathrm{KOH}$, as shown in Figure 3. In addition, the cycle stability measurement exhibited no detectable distinction after processing 50 and 100 cycles, whereas an excellent on-off behavior was still preserved even after 1 month. Furthermore, the PEC performance of BP nanosheet-based $\mathrm{PD}$ was evaluated in various $\mathrm{KOH}$ concentrations, which demonstrated that the as-prepared BP nanosheets might have a great potential application in self-powered PDs. The present work provides fundamental acknowledgment of the performance of a PEC-type BP nanosheet-based PD, offering extendable availabilities for 2D BP-based heterostructures to construct high-performance PEC devices.

Youngblood et al. [26] demonstrated a waveguide-integrated and gate-tunable BP PD integrated on an Si photonic waveguide operating in the NIR telecom band. In a significant advantage over graphene devices, these BP PDs could operate under bias with very low dark current and attained
A
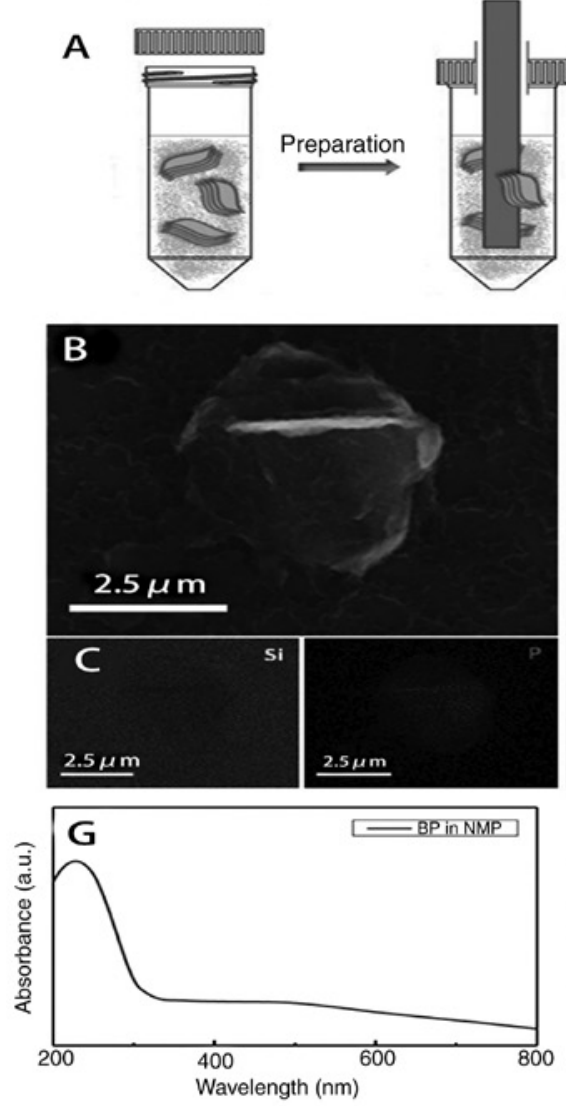
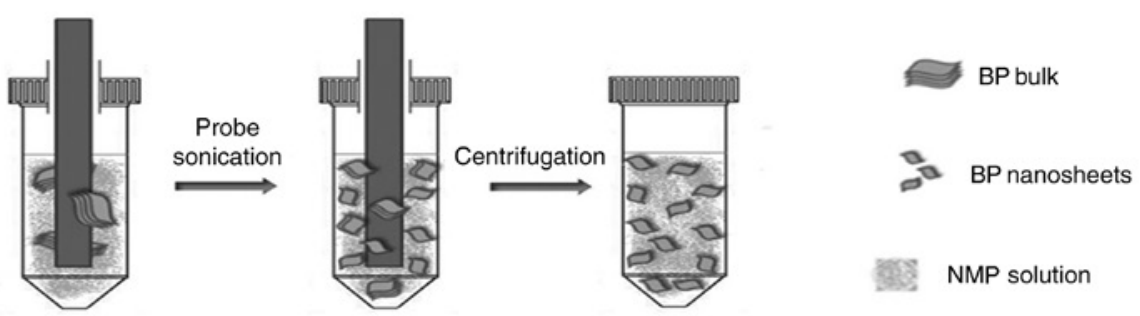

NMP solution
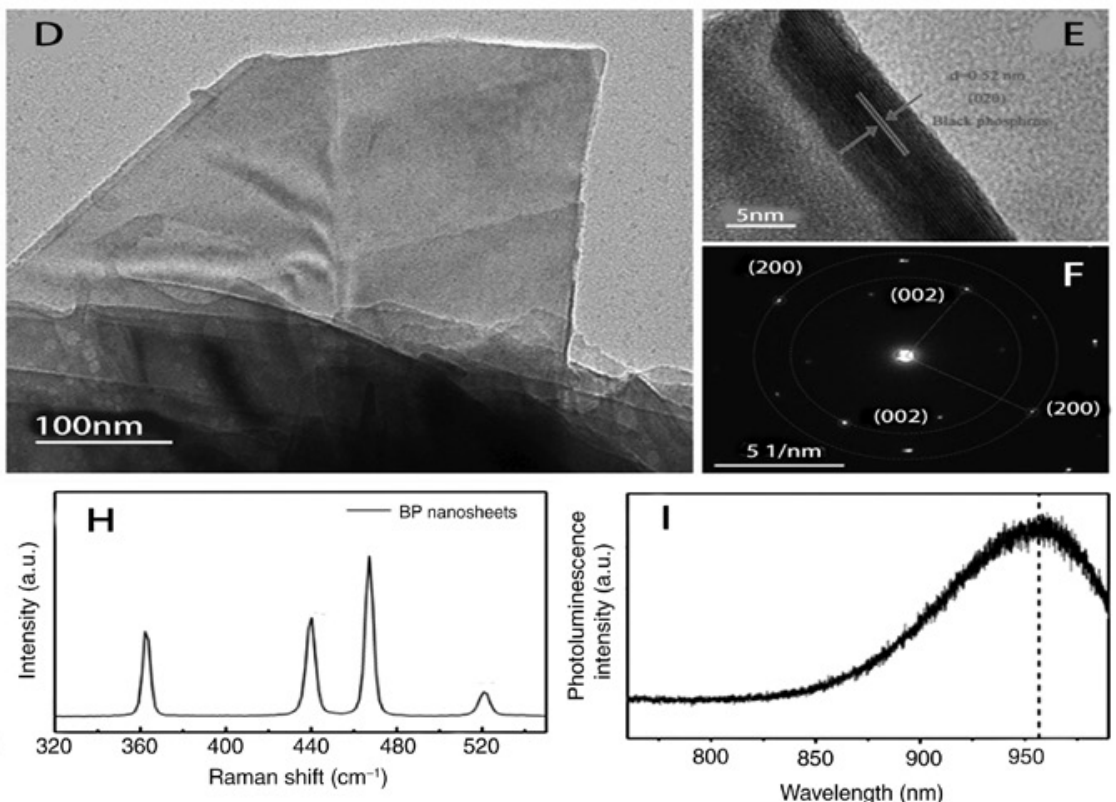

Figure 3: Investigation of BP's optical property. (A) Schematic illustration of the proposed solution-phase exfoliation route for BP nanosheets. (B) Scanning electron microscopy (SEM) image. (C) EDS image. (D) Transmission electron microscopy (TEM) image. (E) High-resolution TEM (HRTEM) image. (F) Selected area electron diffraction (SAED) pattern of exfoliated BP. (G) UV-visible spectra of BP/NMP (inset: optical image of BP/NMP solution). (H) Raman spectra of bulk BP and exfoliated $\mathrm{BP}$ nanosheets on $\mathrm{SiO}_{2} / \mathrm{Si}$ substrate.

(I) Photoluminescence spectra of exfoliated BP nanosheets on $\mathrm{SiO}_{2} / \mathrm{Si}$ substrate. Reproduced with permission from Ref. [26]. 
an intrinsic responsivity up to 135 and $657 \mathrm{~mA} / \mathrm{W}$ in 11.5 and 100-nm-thick devices, respectively, at room temperature. The photocurrent was dominated by the photovoltaic effect with a high response bandwidth exceeding $3 \mathrm{GHz}$.

Deng et al. [27] demonstrated a gate-tunable p-n diode based on a p-type BP/n-type monolayer $\mathrm{MoS}_{2}$ van der Waals $p$-n heterojunction. Upon illumination, these ultrathin $\mathrm{p}$-n diodes showed a maximum photodetection responsivity of $418 \mathrm{~mA} / \mathrm{W}$ at the wavelength of $633 \mathrm{~nm}$ and photovoltaic energy conversion with an external quantum efficiency of $0.3 \%$. These $\mathrm{p}$-n diodes show promise for broadband photodetection.

Miao et al. [28] demonstrated a truly vertically stacked and self-encapsulated van der Waals heterojunction diodes built from 2D semiconductors ( $\mathrm{MoS}_{2}$ and $\left.\mathrm{BP}\right)$ and compared its performance to conventional lateral 2D heterojunction devices (partially overlapped 2D heterostructures), as shown in Figure 4. Both vertical and lateral p-n heterostructure diodes exhibited a strong rectification ratio without gate voltage applied. Moreover, the current density under forward bias vertical diode delivered 70 times higher than a conventional lateral device design; it is attributed to the complete elimination of series resistance. This work demonstrates the potential of using the vertically stacked 2D semiconductors for future nanoelectronic and optoelectronic devices with optimal performance.

Li et al. [29] first fabricated a bipolar phototransistor based on WSe ${ }_{2}$-BP-MoS 2 van der Waals heterostructure. Broadband photoresponsivities for visible $(532 \mathrm{~nm})$ and IR $(1550 \mathrm{~nm})$ light waves reach up to 6.32 and $1.12 \mathrm{~A} / \mathrm{W}$,

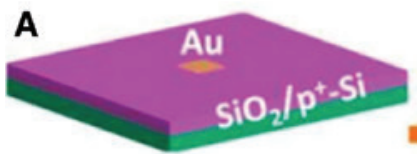

(i) Bottom Au electrode

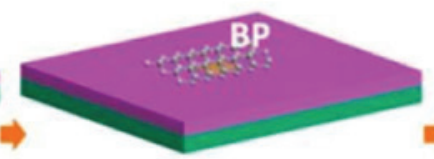

(ii) $\mathrm{BP}$ on Au electrode

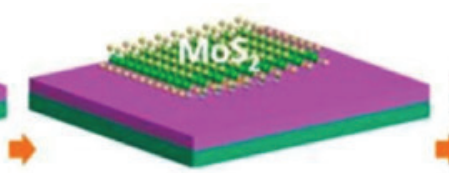

(iii) $\mathrm{MoS}_{2}$ on BP flake
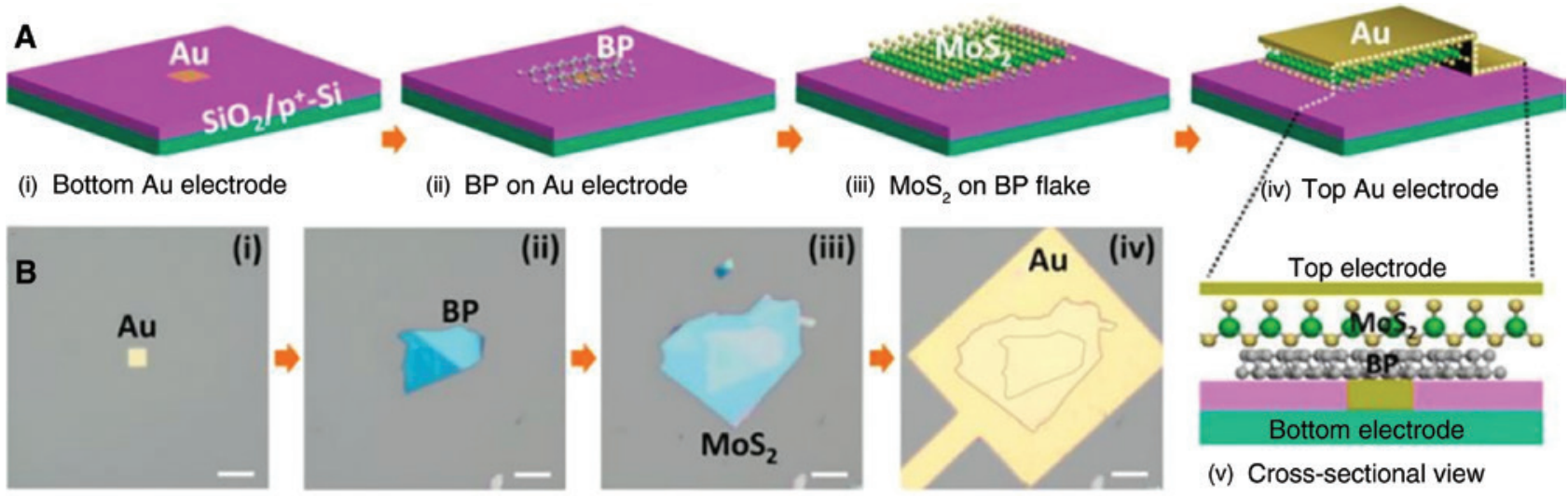

(v) Cross-sectional view
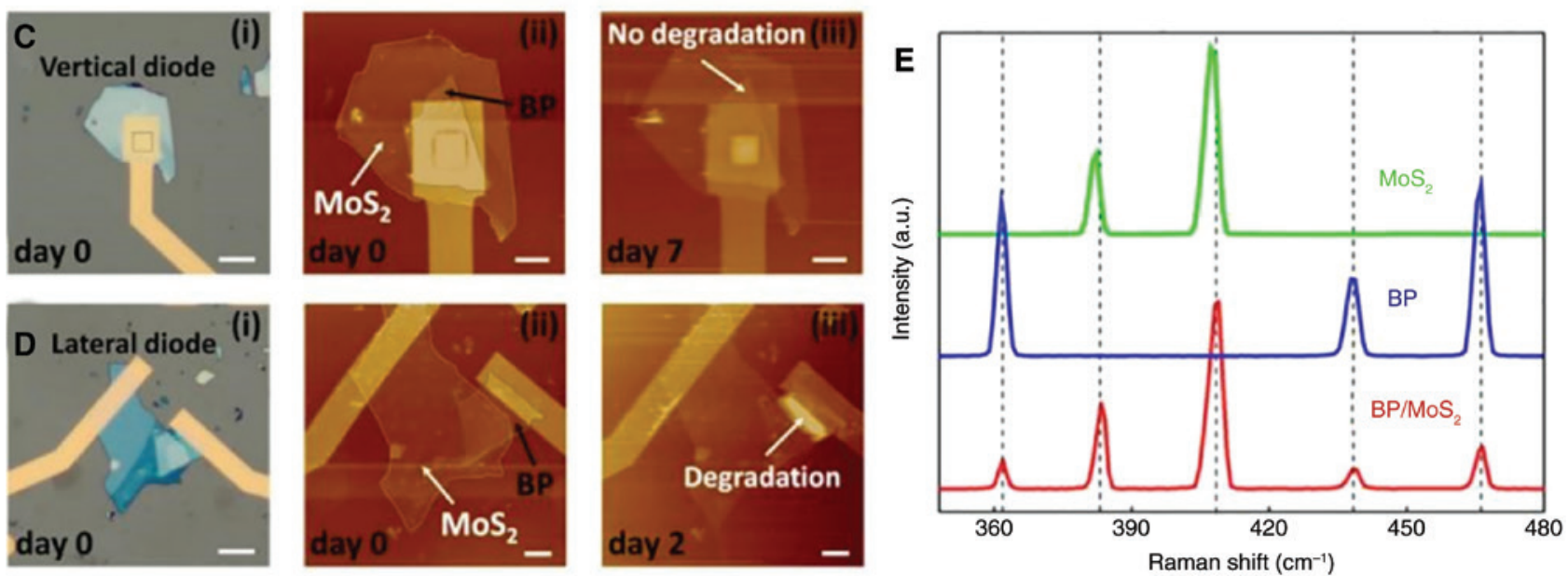

Figure 4: Fabrication procedure and characterization of vertically and laterally stacked $B P / \mathrm{MoS}_{2}$ heterojunction $\mathrm{p}$-n diodes. (A) Schematic diagrams illustrating the fabrication procedure of vertically stacked $B P / M o S_{2} p$-n diodes. A $50 \mathrm{~nm}$-thick Au film was used to fill the square trench followed by lift-off to form the bottom electrode (A-i). Fewlayer $\mathrm{p}$-type BP flake was then transferred onto the as-fabricated $\mathrm{Au} / \mathrm{p}^{+}-\mathrm{Si}$ bottom electrode (A-ii). Next, the $\mathrm{MoS}_{2}$ flake fully covers the BP (A-iii). Finally, the top electrode was patterned onto the MoS ${ }_{2}$ flake by EBL and thermal evaporation of $50 \mathrm{~nm}$-thick Au film (A-iv). The current can flow between the bottom Au/p+-Si electrode and the top Au electrode through the semiconducting $\mathrm{BP} / \mathrm{MoS}_{2}$ heterostructure (A-v). (B) Optical microscope images showing a representative vertical BP/MoS 2 p-n junction diode after each fabrication step including $A u(B-i), B P(b-i i), M o S_{2}$ (B-iii) and top Au electrode (B-iv). Scale bar, $10 \mu \mathrm{m}$. (C and D) Ambient stability study of (C) vertically and (D) laterally stacked BP/MoS diodes. Scale bar, $10 \mu \mathrm{m}$ (C-i and D-i) and $5 \mu \mathrm{m}$ (C-ii, C-iii, D-ii, and D-iii). (E) Raman spectra of $\mathrm{MoS}_{2}, \mathrm{BP}$, and BP/MoS heterostructure. Reproduced with permission from Ref. [29]. 
respectively, which are both improved by tens of times in comparison to similar photodiode devices composed of $\mathrm{WSe}_{2}$-BP. The phototransistor also exhibited ultrasensitive shot noise limit specific detectivities, which were $1.25 \times 10^{11}$ Jones ( 1 Jones $=1 \mathrm{~cm} \mathrm{~Hz}^{1 / 2} \mathrm{~W}^{-1}$ ) for visible light at wavelength $\lambda=532 \mathrm{~nm}$ and $2.21 \times 10^{10}$ Jones for the NIR light at wavelength $\lambda=1550 \mathrm{~nm}$ at room temperature. This generic strategy by combining 2D layered materials into heterostructures to enable multifunctional devices and components may open a new avenue for high-density monolithic integration of functional devices in a limited area.

Ding et al. [30] constructed a novel 2D van der Waals heterostructure made by InSe and BP monolayer with high hole mobility $\left(10^{3} \mathrm{~cm}^{2} \mathrm{~V}^{-1} \mathrm{~s}^{-1}\right)$ and investigated its structural and electronic properties using first-principles calculations. The InSe/BP heterostructure could be formed easily due to its negative binding energy. Its heterostructure exhibited an intrinsic type II band alignment with a direct bandgap of $1.39 \mathrm{eV}$, in which electrons were located in the InSe layer and hole localized in the BP layer. The band offsets of InSe and BP are $0.78 \mathrm{eV}$ for CBM and $0.86 \mathrm{eV}$ for VBM, respectively, which greatly enhance the performance of InSe-based photovoltaic devices and PDs. The electron mobility of the InSe/BP heterostructure is increased to $3 \times 10^{3} \mathrm{~cm}^{2} \mathrm{~V}^{-1} \mathrm{~s}^{-1}$ and hole mobility is increased from $40 \mathrm{~cm}^{2} \mathrm{~V}^{-1} \mathrm{~s}^{-1}$ in InSe monolayer to $10^{4} \mathrm{~cm}^{2} \mathrm{~V}^{-1} \mathrm{~s}^{-1}$, which is three orders of magnitude larger than that in InSe monolayer. Thus, the InSe/BP heterostructure is considered to have a huge potential application in PDs.

Ye et al. [31] demonstrated a PD with visible to NIR detection range based on the heterojunction fabricated by van der Waals assembly between few-layers BP and fewlayers $\mathrm{MoS}_{2}$. The heterojunction with electrical characteristics, which could be electrically tuned by a gate voltage, achieves a wide range of current-rectifying behavior with a forward-to-reverse bias current ratio exceeding $10^{3}$. The photoresponsivity of the PD was about $22.3 \mathrm{~A} / \mathrm{W}$ measured at $\lambda=532 \mathrm{~nm}$ and $153.4 \mathrm{~mA} / \mathrm{W}$ at $\lambda=1.55 \mu \mathrm{m}$ with a microsecond response speed $(15 \mu \mathrm{s})$. In addition, its specific detectivity was calculated to have the maximum values of $3.1 \times 10^{11}$ Jones at $\lambda=532 \mathrm{~nm}$ and $2.13 \times 10^{9}$ Jones at $\lambda=1550 \mathrm{~nm}$ at room temperature. This generic strategy to integrate 2D layered materials in the vertical direction to enable functional devices and circuits may open up a new dimension for high-density integration of functional devices in the limited circuit area.

In addition, BP can also be configured as an excellent UV PD. Wu et al. [32] investigated a UV PD based on BP with a specific detectivity of $3 \times 10^{13}$ Jones for the first time. The UV photoresponsivity could be significantly enhanced to $9 \times 10^{4} \mathrm{~A} / \mathrm{W}$ by applying a source-drain bias of $3 \mathrm{~V}$, which was the highest ever measured in any 2D materials and $10^{7}$ times higher than previously reported value for BP. Such a colossal UV photoresponsivity was attributed to the resonant-interband transition between two specially nested valence and conduction bands. These nested bands provide an unusual high density of states for highly efficient UV absorption due to their singularity nature. This work shows that BP proves to be a strong viable candidate for future optoelectronic applications especially as state-of-the-art UV detectors.

\section{1D nanostructured PDs}

\section{1 $\mathrm{ZnO}$ nanowire film}

$\mathrm{ZnO}$ is a group II to VI wide-bandgap semiconducting metal oxide with high electron mobility and exciton binding energy, which makes it an interesting material for a variety of applications [33, 34]. Owing to their wide bandgap (3.4 eV) [35], high exciton binding energy (60 MeV), and high photoconductive characteristic, $\mathrm{ZnO}$ nanostructures are frequently used to construct UV PDs [36-39]. ZnO films with high specific area and physical flexibilities are easy and cheap to prepare and process with low costs, and the photoconductivity of $\mathrm{ZnO}$ film PDs changes notably upon on-off state switching, demonstrating their potential in practical applications [37, 40]. In particular, forming heterojunctions between $\mathrm{ZnO}$ and other materials [ZnS, poly(vinyl alcohol), poly(acrylonitrile), and poly(vinylcarbazole)] has significant effects on improving the photoelectric performance of $\mathrm{ZnO}$ film-

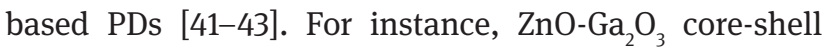
microwire $\mathrm{PD}$ is highly suitable for practical applications of solar-blind photodetection [44].

A novel $\mathrm{CdMoO}_{4}-\mathrm{ZnO}$ composite film has been prepared by spin-coating $\mathrm{CdMoO}_{4}$ microplates on $\mathrm{ZnO}$ film and was constructed as a heterojunction PD. With the aid of $\mathrm{CdMoO}_{4}$ microplates, the structure of the PD changed from MSM type to a heterojunction one, which largely benefited the photoelectric performance. Ouyang et al. [37] deposited $\mathrm{Au}$ nanoparticles (NPs) to modify the $\mathrm{CdMoO}_{4}^{-}$ $\mathrm{ZnO}$ composite film, and compared to the CMO-ZnO PD, this PD achieves a higher photocurrent of $289.1 \mathrm{nA}$ and a higher responsivity of $321.1 \mathrm{~mA} / \mathrm{W}$. At the same time, its decay time decreases to $9.2 \mathrm{~s}$. This further improvement of photoelectric properties was due to the hot electron injection effect of AuNPs and the Schottky barriers formed between the AuNPs and CMO-ZnO composite film block the carrier transportation. However, an excess loading 
of $\mathrm{CdMoO}_{4}$ microplates might shelter the $\mathrm{ZnO}$ film underneath and block the light absorption of the composite film, which would lead to the decrease of photocurrents. Thus, in practical applications, it should be avoided. The study on Au-CMO-ZnO composite film PD might be potentially useful for facile construction of $\mathrm{ZnO}$-based PD with novel structure and high photoelectric performance.

Teng et al. [42] incorporated a $\mathrm{BiOCl}$ nanostructure into the $\mathrm{ZnO}$ film and constructed them as film PDs. With forming barriers to block the straight transmission of electrons between electrodes, the rise/decay time of $\mathrm{BiOCl} /$ $\mathrm{ZnO}$ hybrid film PD was 25.83/11.25 s, which was much shorter than that of pure ZnO film PD (69.34/>120 s). Due to the injection of photogenerated electrons into the $\mathrm{ZnO}$ film under UV light illumination, the responsivity of the BiOCl/ZnO hybrid film PD in the UV region could reach $182.87 \mathrm{~mA} / \mathrm{W}$, which was about 6.87 times that of pure $\mathrm{ZnO}$ film PD. This is different from those methods that enhanced the performance of PDs by adjusting the depletion layer on the surface of $\mathrm{ZnO} \mathrm{NPs}$. The $\mathrm{BiOCl} / \mathrm{ZnO}$ hybrid structure film PD provides a new and simple route for the enhancement of optoelectronic performance based on the $\mathrm{ZnO}$ nanostructures.

Duan et al. [45] fabricated a $\mathrm{ZnO}: \mathrm{Al} / \mathrm{Si}$ heterojunction $\mathrm{PD}$ by a simple and low-cost CBD method. $\mathrm{ZnO} \mathrm{Al} / \mathrm{Si} \mathrm{PD}$ showed a sharp cutoff responsivity at $400 \mathrm{~nm}$, a UV-tovisible rejection ratio $\left(R_{386 \mathrm{~nm}} / R_{500 \mathrm{~nm}}\right)$ of 50 , and fast rise and decay times of the photoresponse, which indicated that the hexagonal nanocrystal (NC) $\mathrm{ZnO}$ :Al film was the most promising materials for the fabrication of high-performance UV PDs. Self-powered UV PDs, which have vast applications in the military and for civilian purposes, have become particularly attractive in recent years due to their advantages of high sensitivity, ultrasmall size, and low power consumption. This should generate extensive interest in this field and encourage more researchers to engage in and tackle the scientific challenges [46-48]. In 2017, Yu et al. [49] fabricated three novel heterojunctions by depositing films through one-step in situ polymerization in acid solution, which provides a simple and efficient approach to prepare high-performance self-power UV PDs. Hu et al. [39] developed a high-performance UV $\mathrm{PD}$ based on an Se/ZnO p-n heterojunction. This device was theoretically equal to a parallel-connection circuit for its special structure, and due to its parallel connection of $\mathrm{ZnO}$ and the Se/ZnO hybrid structure, this UV PD realized a binary response of positive and negative output currents using an on-off light source at a small reverse bias. Additionally, the speed of the ZnO UV PD is greatly improved with a rise time of $0.69 \mathrm{~ms}$ and a decay time of $13.5 \mathrm{~ms}$, which are much quicker than those of pure $\mathrm{ZnO}$ film PDs.
All the results demonstrate that the high-performance UV PD based on an Se/ZnO p-n self-powered heterojunction provides a promising way to fabricate $\mathrm{ZnO}$-based $\mathrm{p}$-n junction UV PDs with high sensitivity, high SNR, high spectral selectivity, high speed, and high stability and thus may lay a solid ground for the future applications of this kind of high-performance multifunctional PD.

Purusothaman et al. [50] synthesized highly crystallized $\mathrm{ZnO}$ microarchitectures ( $\mathrm{ZnO}$ microwire, $\mathrm{ZnO}$ corallike microstrip, and $\mathrm{ZnO}$ fibril-like clustered microwire) through a simplified vapor transport technique (carrier gas/catalyst-free). As-fabricated PD is shown in Figure 5. Among them, $\mathrm{ZnO}$ fibril-like clustered microwire exhibited higher IPh290 $\mu \mathrm{A}$ at $17 \mathrm{~mW} \mathrm{~cm}{ }^{-2}$ and comparable responsivity $\left(R_{405 \mathrm{~nm}}\right)$ of $716 \mathrm{~mA} / \mathrm{W}$. In contract to reported microarchitectures, $\mathrm{ZnO}$ fibril-like clustered microwire exhibited superior photosensing behavior with a detectivity range of $6.72 \times 10^{10}$ Jones and broadened spectral activity extending from UV to the visible region $(365,405$, and $535 \mathrm{~nm}$ ). The results reported in this paper provide a facile route to develop flexible self-powered optical communication systems and sensors.

Chen et al. [51] developed a strain modulated solarblinded avalanche PD based on $\mathrm{ZnO}-\mathrm{Ga}_{2} \mathrm{O}_{3}$ core-shell heterojunction microwire. This PD is highly sensitive to deep UV light centered at $261 \mathrm{~nm}$. Due to its ultrahigh sensitivity and spectral selectivity, this PD could respond to rare weak deep UV light with almost no response to visible light. Moreover, the detection could be enhanced by applying certain static strains on the device through the piezo-phototronic effect. The current response could be enhanced about three times under $-0.042 \%$ strain. The strain-induced piezopotential modulates carrier transport mainly in the $\mathrm{ZnO}$ core. The photoresponse of this PD was enhanced by this effect and thus made it a more perspective PD in human-machine interaction areas, such as health monitoring, smart systems, and security communication.

\subsection{CNTs}

1D semiconductor nanostructures have stimulated much attention in high-performance PDs, owing to the pronounced surface effects originating from the large surfaceto-volume ratio [52, 53]. Since the discovery of multiwalled CNT by Lijima and Ichihashi and the related single-walled CNTs in 1993 [54], CNTs exhibited some strong advantages for applications in IR detectors in comparison to other 1D candidate materials, e.g. the naturally nanoscale size, simplified growth and device fabrication, chirality-dependent 

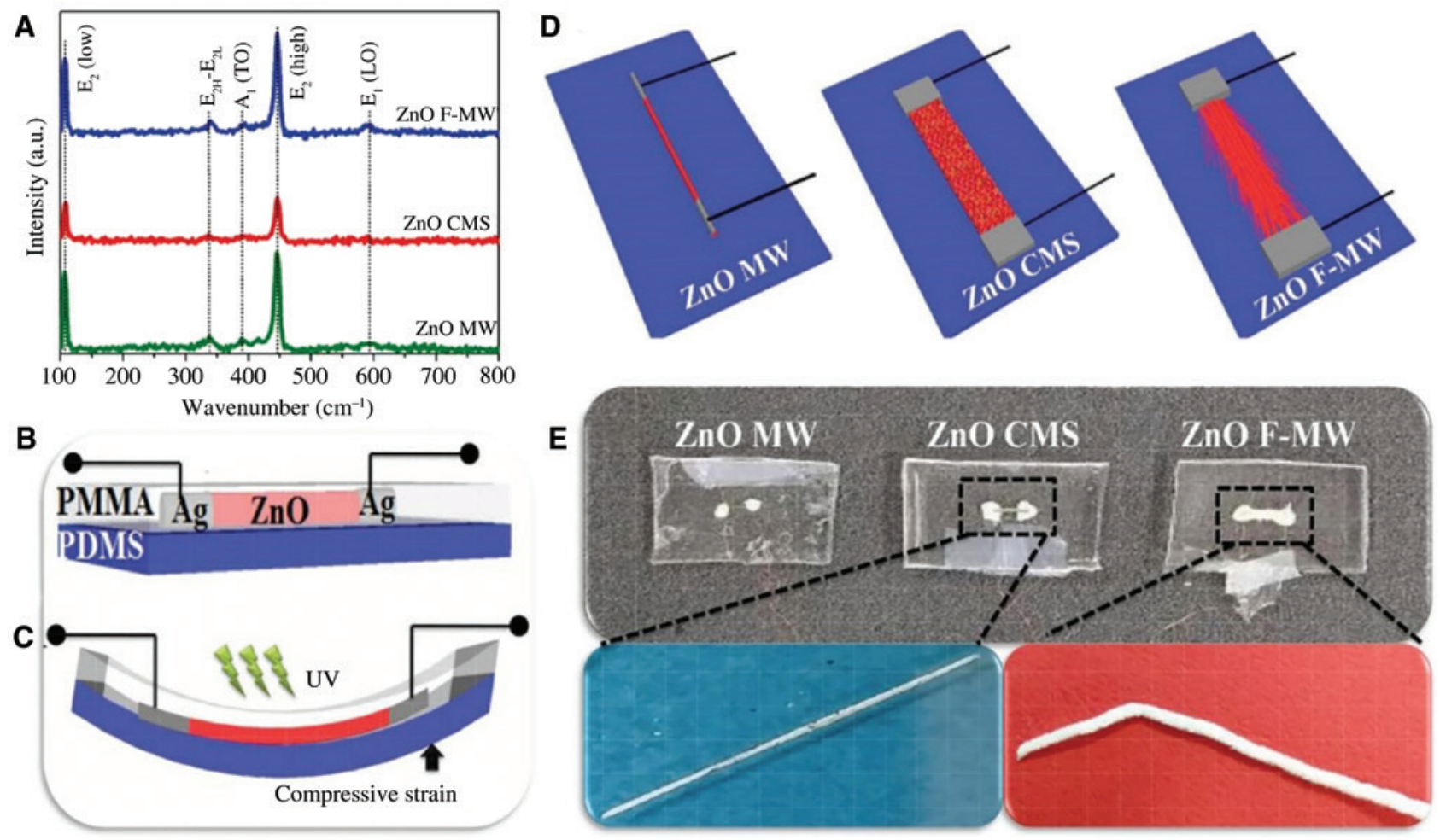

Figure 5: Structure and photospectrum of this as-fabricated ZnO. (A) Rama spectrum of ZnO microarchitectures. (B) Schematic representation of as-fabricated PD. (C) Compressive strain-induced behavior of PD. (D) Schematic view of ZnO device architectures. (E) Optical images of as-fabricated PD devices using ZnO MW, ZnO CMS, and ZnO F-MW. Reproduced with permission from Ref. [50].

direct band structures that cover important communication wavebands, and room-temperature operation due to their moderate bandgap energies ( $\gg 26 \mathrm{meV})$ [55]. The very small size and good extendibility also provide great prospects for their flexible integrations into other photonic or hybrid optoelectronic systems [56, 57].

However, for CNT-based near- or shortwave-IR optoelectronics, the existing developments for enhanced absorption or signal detection are insufficient [58]. Besides, for signal sensing or information imaging applications, which involve the detection of a specific target signal among a broad background of signals or noise, the photoresponse of CNT aggregations usually covers a wide NIR scope with various characteristic peaks induced by chirality-dependent Van Hove singularity transitions [59], leading to random responses without any targets. That is to say, a specific response to a target signal requires a higher suppression ratio [60]. To further increase the signal outputs, the hybrid systems that enhance light absorption can also be considered. Compared to the fast progress in the fields of 2D materials [61], some concepts for enriching CNT PDs should be further investigated and developed. Here, we present a figure of merit and some enhanced light absorption PDs in this section.
In 2016, Liang et al. [55] demonstrated a high photoelectric conversion efficiency and signal recognition CNT PDs by integrating with a designed F-P cavity. A 6-fold enhanced optical absorption could be achieved because of the confined effect of the designed optical mode, and the detectors exhibited a higher suppression ratio until a power density of $0.07 \mathrm{~W} \mathrm{~cm}^{-2}$ and photocurrent of $5 \mathrm{pA}$, and the spectral full-width at half-maximum is $\sim 33 \mathrm{~nm}$ at a signal wavelength of $1200 \mathrm{~nm}$. More importantly, a concept of the "resonance and off-resonance" cavity was developed at signal detection at zero bias, which opened the possibility of chirality-specific CNT film-based signal recognition systems for information photonics on a chip and broadening the potential for the application of other nanomaterials in information photonics regardless of their doping polarity. The concept offered a promising and practical pathway for the development of stable, largescale, multiwavelength signal capture, recognition, and sensing systems.

Pyo et al. [62] demonstrated a heterogeneous all-carbon PD composed of graphene electrodes and porphyrin-interfaced single-walled CNTs channel, exhibiting high photoresponse, flexibility, and full transparency across the device. The porphyrin molecules generate 
and transfer photoexcited holes to the single-walled CNTs even under weak white light, resulting in significant improvement of photoresponsivity from negligible to $1.6 \times 10^{-2} \mathrm{~A} \mathrm{~W}^{-1}$. Simultaneously, the PD exhibited high flexibility, allowing stable light detection under $\approx 50 \%$ strain (i.e. a bending radius of $\approx 350 \mu \mathrm{m}$ ) and retaining a sufficient transparency of $\approx 80 \%$ at $550 \mathrm{~nm}$. Notably, minimal loss in photoresponse even after heavy crumpling (a strain of $\approx 50 \%$ ) and ability to detect sunlight gave the device great potential as an epidermal sunlight sensor. This PD could be used for low-cost, large-scale, and high-performance optoelectronic applications that require mechanical flexibility and optical invisibility. The hybridization of low-dimensional nanomaterials envisions a promising strategy for achieving flexible and transparent electronics as well as enabling further exploration into the unprecedented functionalities of nanomaterials.

However, the absorption restriction from single layer limits the effective utilization of incident light. In 2017, Huang et al. [63] demonstrated a plasmonic electrode structure in CNT thin-film PD based on randomly deposited high-purity semiconducting CNT, which could collect photoinduced carriers effectively and enhance light absorption at the same time. The plasmonic enhancement resonate around $1650 \mathrm{~nm}$ wavelength well corresponding to our simulation, which was a short-wave IR for plasmonic enhancement application. Furthermore, the best performance improvement of CNT detector with plasmonic structure could be enhanced as 13.7 times for photocurrent mode and 5.62 times for photovoltage mode than those devices without structure at $1650 \mathrm{~nm}$ resonant wavelength. At last, the plasmonic structures were applied on tandem PDs with nine virtual contacts, and both the photocurrent and the photovoltage were increased. By further improving the structure and the quality of CNT film, such as dense semiconducting CNTs array with thicker layer, the plasmonic detectors will show more powerful use in modern room temperature IR detection and even in optical integration circuit with compact construction.

\section{OD PbS QDs}

$\mathrm{PbS}$ has been among the most intensively investigated as a candidate material for solution-processed optoelectronics $[64,65]$. This is primarily due to its small bandgap and large Bohr exciton radius, which collectively give it wide spectral tunability in the IR [66]. Recent efforts employing micron-sized ultrathin 2D PbS nanoplates as the active material in PD devices showed efficient detection in the IR [67]. However, these nanoplates were fabricated via a chemical vapor deposition process that can be expensive and challenging to scale up in terms of production volume [68]. In 2010, Schliehe et al. [69] introduced the wet-chemical synthesis of $2 \mathrm{D} \mathrm{PbS}$ nanosheets based on the $2 \mathrm{D}$ oriented attachment of sphere-like $\mathrm{PbS}$ particles into ultrathin planar structures. Their approach was subsequently adopted by other researchers who explored different synthesis parameters that allowed for shape and thickness control. Aside from oriented attachment, nucleation and growth within a lamellar noctylamine bilayer template has also been shown to produce pseudohexagonal $\mathrm{PbS}$ quantum plates. Despite theoretical predictions of improved carrier mobility and stronger photon absorption [70], a thin film of nanosheets produced via the method of Schliehe et al. [68] yielded relatively low responsivity when used within a PD configuration. Additionally, we found that these nanosheets are prone to severe aggregation upon processing and cannot easily be separated into isolated sheets even after prolonged sonication. These limitations prompted us to develop an alternative approach to the synthesis of 2D PbS NCs. Here, a new solutionprocessed PbS QD was fabricated and applied for PDs.

Wu et al. [67] reported the synthesis of colloidal 2D PbS nanoplates with residual $\mathrm{Cu}_{2} \mathrm{~S}$ domains via a partial cation-exchange process involving $\mathrm{Pb}^{2+}$ and presynthesized hexagonal $\mathrm{Cu}_{2} \mathrm{~S}$ nanoplates with an average thickness of $3 \mathrm{~nm}$ and edge lengths of $150 \mathrm{~nm}$. Due to the low resistivity of $8.04 \Omega \mathrm{cm}$ of the PbS nanoplate film, which was comparable to commercial doped semiconductors, the responsivity and specific detectivity at $808 \mathrm{~nm}$ was $1739 \mathrm{~A} / \mathrm{W}$ and $2.55 \times 10^{11}$ Jones, respectively. This work uses partial cation exchange and plate-like morphology in colloidal PbS NC film to improve its in-plane conductivity while retaining good photoresponse, and it needs to be investigated in much more detail how better photodetection efficiencies may be expected with higher NC packing densities and a more judicious choice of ligands, making these heterostructured PbS NPL films possible candidates for low-cost high-performance solution-processed NIR PDs.

Shen et al. [71] demonstrated a highly sensitive, visible-blind, and narrow-band NIR PD with a full-width at half-maximum of $50 \mathrm{~nm}$ and a visible rejection ratio of 100. This device had a thick nanocomposite absorbing layers made of PbS QDs, which effectively enhanced both the responsivity and the visible rejection ratio of external quantum efficiency by 10 -fold compared to those without $\mathrm{PbS}$ QDs. Encouragingly, the inclusion of $\mathrm{PbS}$ QDs does not increase the device noise. We directly measured a noise equivalent power of $6.1 \mathrm{pW} \mathrm{cm}{ }^{-2}$ at $890 \mathrm{~nm}$ 
and a large linear dynamic range of more than 11 orders of magnitude. The highly sensitive visible-blind NIR narrow-band PDs may find applications in biomedical engineering.

Lee et al. [72] fabricated a solution-processed inorganic UV-visible-SWIR PD with light sensitivity from 350 to $2000 \mathrm{~nm}$ using highly monodispersed large $\mathrm{PbS}$ NCs with extremely low size dispersions (5\%). These highly monodispersed large PbS NCs were synthesized by multiple injection of the sulfur precursor solution to grow the NCs without initiating new nucleation during growth, which enabled PbS NC synthesis with excellent particle size control and resulted in well-defined absorption peaks at long wavelengths. This UV-visible-SWIR multispectral PD showed detectivity values of more than $1 \times 10^{11}$ Jones from 350 to $2000 \mathrm{~nm}$ and a maximum detectivity value of $1.2 \times 10^{12}$ Jones at the peak wavelength of $1800 \mathrm{~nm}$. This work realized the synthesis of large PbS NCs with low cost and practical applications.

Qiao et al. [73] demonstrated spectra-selective PbS QD IR PDs using the semiconductor's “filter" absorption characteristics and QD quantum size effect. Different from the wide detection spectra of traditional PDs, this $\mathrm{PbS}$ QD PDs can detect the spectra-selective light source. Spectra-selective PDs of perovskite/QDs and QDs/QDs were respectively implemented by integrating two functional layers. The top layer (facing the light) was used to filter the nontarget spectra and the bottom layer was used for detecting. When tuning the QD size, the response spectrum wavelength and range of spectra-selective PDs could be conveniently tailored. The obtained selectivity factor and normalized detectivity ratio from target and nontarget illumination can reach at least 10 . These favorable properties promised spectra-selective PDs to be widely applied in identifying an unknown light source and future artificial recognition, artificial intelligence, communication, and surveillance system.

Mishra et al. [74] fabricated NIR PDs with a very simple architecture comprising a $\mathrm{PbSe} / \mathrm{PbS}$ tetrapod layer between two Au electrodes on a glass substrate. $\mathrm{PbSe} / \mathrm{PbS}$ tetrapod was synthesized by the cation exchange method. Due to its branched morphology, when illuminated by an NIR laser, these devices achieved a responsivity of $11.9 \mathrm{~A} / \mathrm{W}$ without the use of ligand-exchange processes, thermal annealing, or hybrid device architecture. This heterostructured $\mathrm{PbSe}$ seeded $\mathrm{PbS}$ nanotetrapods was the most promising material of NC-based NIR PD. More importantly, the use of cationic exchange processes to derive anisotropic semiconductor nanoheterostructures can result in a fortuitous combination of properties that may be highly desirable for solution-processed optoelectronic applications.
Lee et al. [75] demonstrated high gain and low dark current solution-processed colloidal PbS QDs IR PIN PDs with IR sensitivity up to $1500 \mathrm{~nm}$. At a peak wavelength of $1200 \mathrm{~nm}$, the PD had a gain of 187 , a detectivity of $7.0 \times 10^{13}$ Jones, and a response time of $3.0 \pm 0.2 \mathrm{~ms}$. Due to its higher detectivity than commercial InGaAs PD, the sufficient response time for imaging applications, and the potentially low-cost fabrication process, this kind of IR PDs was promising for low-cost IR sensing applications [52].

Recently, a new kind of semiconductor, halide perovskite, has attracted worldwide attention. Intrinsically high mobility and large absorption coefficient endow halide perovskites with great promise for high-performance PDs [76-78]. Liu et al. [79] demonstrated solution-processed ultrasensitive broadband $\mathrm{PDs}$ using $\mathrm{CH}_{3} \mathrm{NH}_{3} \mathrm{PbI}_{3}$ and $\mathrm{PbS}$ QDs as the light harvesters. When passivating the structural defects on the surface of PbS QDs with diminutive molecular-scaled $\mathrm{CH}_{3} \mathrm{NH}_{3} \mathrm{PbI}_{3}$, the dark current of the $\mathrm{PbS} \mathrm{QDs} / \mathrm{CH}_{3} \mathrm{NH}_{3} \mathrm{PbI}_{3}$-based PDs were tremendously suppressed, which effectively brought down the recombination losses and consequently raised the photoresponse of the PDs. As a result, PDs exhibited a broad spectral response from 375 to $1100 \mathrm{~nm}$, with high responsivity of more than 300 and $130 \mathrm{~mA} / \mathrm{W}$ and high detectivity larger than $10^{13}$ and $5 \times 10^{12}$ Jones in the visible and NIR regions. The results offered a facile and promising route for advancing the performance of broadband PDs.

However, when integrating PDs with metal NPs, the improvement of the overall device performance is severely hampered. In 2017, Ren et al. [80] fabricated a bilayer QD-only PD. Due to the QD junction controlled photocarrier dynamics, upon light on, the junction greatly facilitates carrier separation at the interface and, upon light off, the junction promotes the recombination of accumulated carriers in the two films via the interface. The bilayer device is superior over the conventional single-layer devices with faster light response, broader linear dynamic range, larger detectivity, and higher SNR along with comparable responsivity. In addition, the bilayer device was successfully fabricated on flexible PI substrate with comparable performance to the glass-based one, signaling great potential of the QD-based bilayer PDs in future flexible optoelectronics.

Wen et al. [81] developed a high-performance IR PD by integrating nonlayered $\mathrm{PbS}$ and layered $\mathrm{MoS}_{2}$ nanostructures via van der Waals epitaxy. The morphology is shown in Figure 6. Density functional theory calculations indicated that $\mathrm{PbS}$ nanoplates were in contact with $\mathrm{MoS}_{2}$ edges through strong chemical hybridization, which was expected to offer a fast transmission path for carriers that enhanced the response speed. The phototransistor 

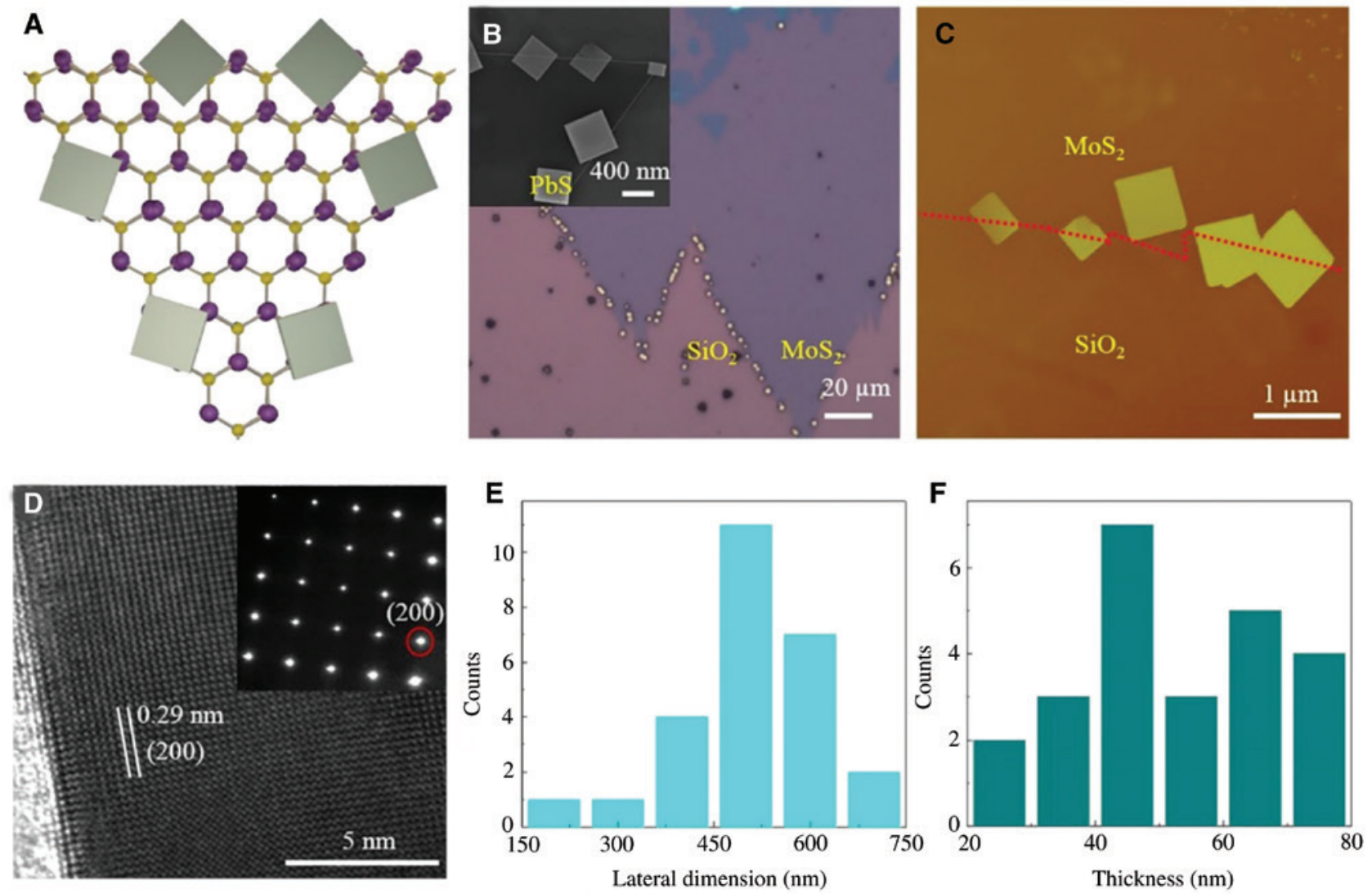

Figure 6: Morphology of nonlayered PbS nanoplates-MoS 2 heterostructures. (A) Schematic illustration of PbS nanoplates-MoS heterostructures. (B) Optical microscopy image with selectively growing PbS nanoplates along the edge of $\mathrm{MoS}_{2}$ triangle. Inset is an amplified SEM image of edge contact heterostructures. (C) Atomic force microscopy image of edge of MoS triangle. (D) HRTEM image with crystal fringes of (200) planes. Inset shows the SAED cubic pattern. (E) and (F) exhibit the statistics of the lateral dimension distribution and thickness distribution of the $\mathrm{PbS}$ nanoplates on $\mathrm{MoS}_{2}$. The histogram statistics of $\mathrm{PbS}$ nanoplates: the lateral dimension and thickness distribution ranging from 150 to 750 and 20 to $80 \mathrm{~nm}$, respectively. Reproduced with permission from Ref. [81].

exhibited a fast response as well as high photoresponsivity $\left(4.5 \times 10^{4} \mathrm{~A} / \mathrm{W}\right)$ and $I_{\text {light }} / I_{\text {dark }}\left(1.3 \times 10^{2}\right)$ in the NIR spectral region at room temperature. In particular, the detectivity was as high as $3 \times 10^{13}$ Jones, which was even better than that of commercial low-layered PDs. Furthermore, by controlling the growth and microfabrication patterning, periodic device arrays of $\mathrm{PbS}-\mathrm{MoS}_{2}$ that were capable of IR detection were achieved on $\mathrm{Si} / \mathrm{SiO}_{2}$ substrates. This work provides a possible method for the integration of PD arrays on Si-based electronic devices and lays a solid foundation for the practical applications of PbS-based devices in the future.

Liu et al. [82] reported solution-processed PDs fabricated by $\mathrm{CH}_{3} \mathrm{NH}_{3} \mathrm{PbI}_{3}$ incorporated with $\mathrm{PbS} \mathrm{QDs}$ through the trap-assisted charge-injection effect. The structure of this device is shown in Figure 7. PbS QDs, which possessed large amount of trap states, were introduced into $\mathrm{CH}_{3} \mathrm{NH}_{3} \mathrm{PbI}$ thin film for establishing ohmic contact at the $\mathrm{CH}_{3} \mathrm{NH}_{3} \mathrm{PbI}_{3} / \mathrm{Al}$ interface, realizing the elimination of the charge injection barrier from the cathode electrode. As a result, an external quantum efficiency of 4500 , a photoresponsivity of $15,000 \mathrm{~mA} / \mathrm{W}$ and a detectivity of more than $6 \times 10^{13}$ Jones at a small bias of $2 \mathrm{~V}$, and a photoresponse time of $11.5 \mu$ s were observed from the solutionprocessed $\mathrm{PDs}$ fabricated by the $\mathrm{CH}_{3} \mathrm{NH}_{3} \mathrm{PbI} 3 \mathrm{PbS} \mathrm{QD}$ nanocomposite thin film.

Owing to their ease of fabrication, low cost, and high flexibility, organic materials have attracted great interest in PD applications. However, suffering from large dark current, small photocurrent, low on-off ratio, and low sensitivity, performances of bare organic-based PDs are not satisfactory. Integrating organic materials with other novel semiconductor materials offers an opportunity to overcome these drawbacks. Wei et al. [83] demonstrated a lateral hybrid organic/PbS QD bilayer PD. Under light illumination, photogenerated electrons and holes are spatially separated via the junction and are transported in the PbS-TbAI QD layer and the organic layer, respectively. 


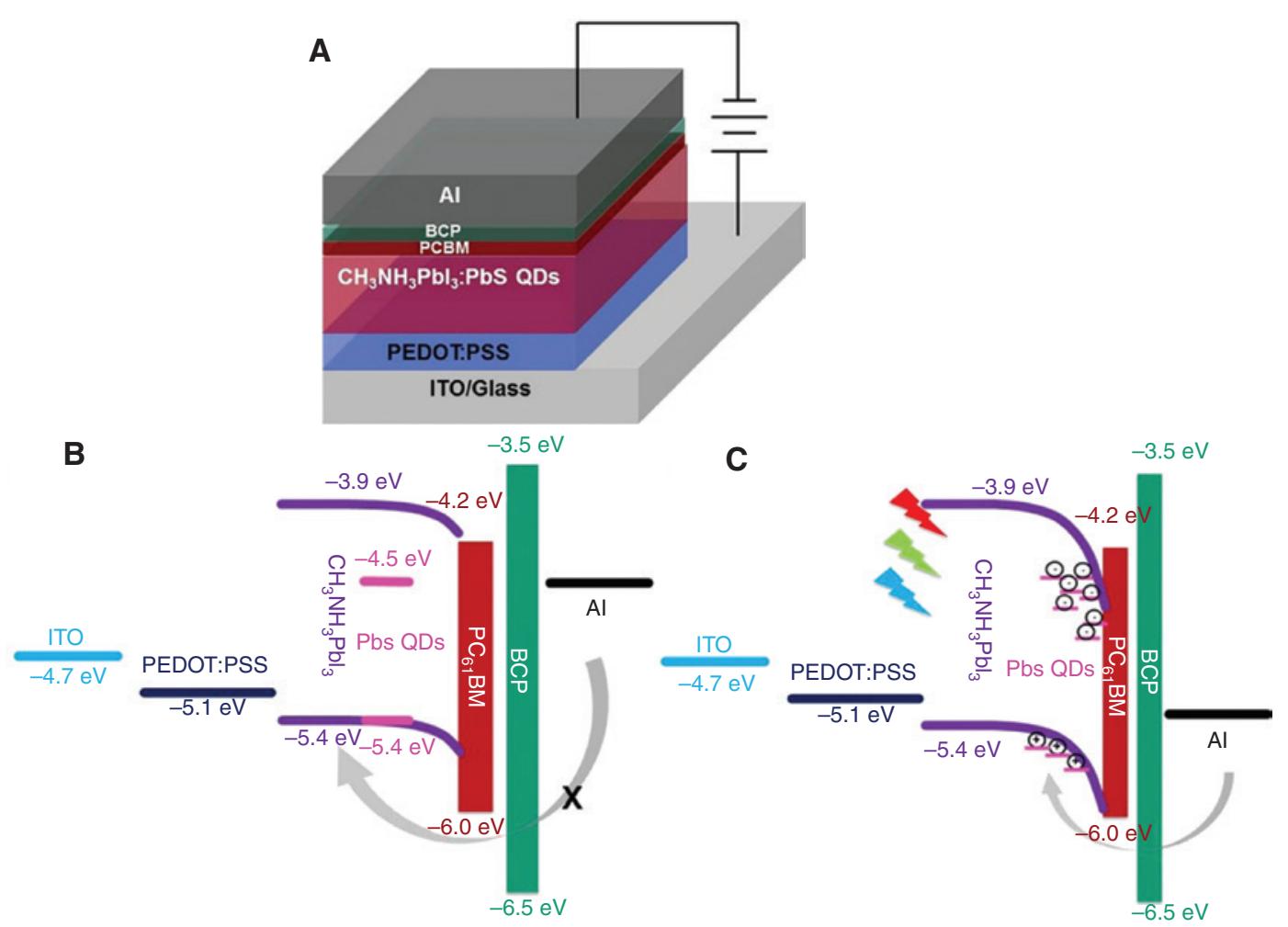

Figure 7: Device structure and proposed working mechanisms of perovskite hybrid PDs (pero-H PDs). (A) Device structure of pero-H PDs and energy level alignment of the materials used for the fabrication of pero-H PDs (B) in dark and (C) under illumination. Reproduced with permission from Ref. [82].

This largely reduced carrier recombination and leads to enhanced photocurrent, and the dark current was greatly suppressed due to the formed organic blend/PbS heterojunction. The bilayer PD significantly outperforms the organic PD and the PbS PD and exhibits high photoresponse of $6.32 \mathrm{~A} / \mathrm{W}$, large SNR of 1690 , and high detectivity of $1.12 \times 10^{13}$ Jones. The bilayer PD fabricated on flexible PI substrate shows comparable performance to the device made on glass substrate, signaling its great potential in future flexible optoelectronics.

\section{Doping}

Most reported PDs are built from inorganic materials such as $\mathrm{ZnO}, \mathrm{CdS}, \mathrm{ZrS}_{2}$, and InAs [84-89]. To improve the performance of PDs, doping techniques are now turning into a sizzling area. Take graphene, for instance; the doping in graphene can be classified into electrical and chemical doping [90]. The doping is referred to as the chemical one when the changes in the graphene lattice structures result via chemical routes, such as substitutional doping with heteroatoms or molecules [91-93]. The goals achieved from such modifications strongly depend on the type of dopants and concentrations and their location within the graphene systems, the chosen sites of graphene for replacement with the foreign impurity atoms, are of critical importance where Fermi level depends on the concentration of doped holes and electrons [83, 94].

Integrating plasmonic nanostructures with graphene demonstrated the possibility of effectively enhancing optical absorption by a strong field enhancement. In 2011, PDs with the AuNP array on graphene, which exhibited $1500 \%$ higher photoresponsivity compared to that without the AuNP array, were reported. The photoresponsivity was plasmonically enhanced by the excited surface plasmons confined in the plasmonic nanostructure. Although the integration enabled high photoresponsivity, most graphene-based PDs with the hybrid approaches still have limited stretchability. Recently, Kim et al. [95] reported a stretchable PD based on the hybrid structure of graphene and graphene QDs with high photoresponsivity. However, their PD has a limited stretchability only up to 25 . Furthermore, mechanical robustness was demonstrated only up to 30 cycles of mechanical stretching.

Ni et al. [96] demonstrate the highly sensitive MIR photodetection of QDs/graphene hybrid phototransistors 
A

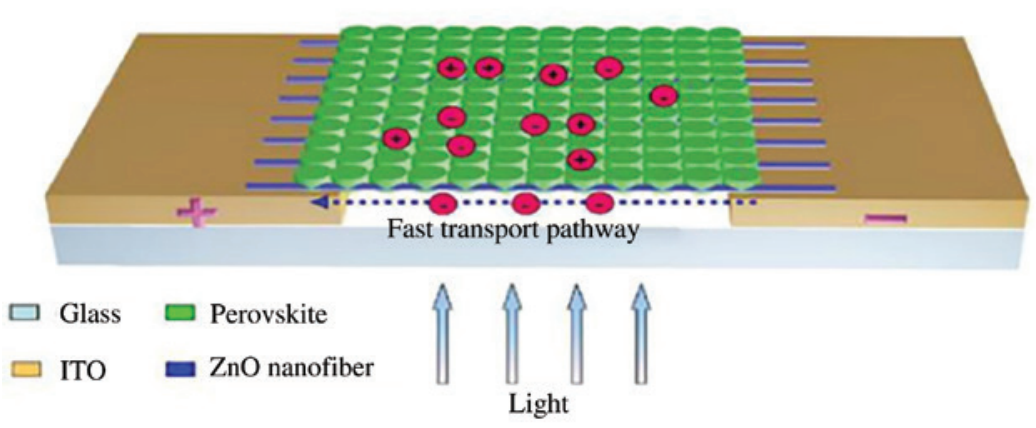

B

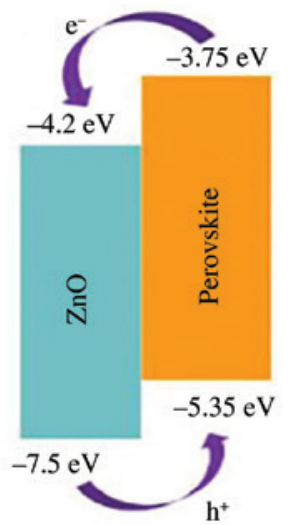

Figure 8: Schematic and band energy of ZnO nanofiber. (A) Schematic of ZnO nanofiber and perovskite hybrid PDs. (B) Band energy alignment between $\mathrm{ZnO}$ and perovskite. Reproduced with permission from Ref. [88].

using plasmonic Si QDs doped with boron. The localized surface plasmon resonance enabled the MIR photodetection of QD/graphene hybrid phototransistors of borondoped Si QDs, and the photogating effect following the band-to-band and sub-bandgap optical absorption of boron-doped Si QDs caused QD/graphene hybrid phototransistors to effectively operate in the UV-to-NIR region. Therefore, ultrabroadband UV-to-MIR photodetection of QD/graphene hybrid phototransistors with high sensitivity was realized. The current work has important implications for the development of high-performance optoelectronic devices based on the hybrid structures of OD plasmonic QDs and 2D materials such as graphene.

Cao et al. [88] fabricated a hybrid PD consisting of electrospun $\mathrm{ZnO}$ nanofibers and perovskites for the first time. As shown in Figure 8, $\mathrm{ZnO}$ nanofibers were synthesized on indium-tin oxide glass with etched gap by the electrospinning method and then the perovskite layer was deposited onto the $\mathrm{ZnO}$ by a solution processing technique. Compared to pristine $\mathrm{ZnO}$ or perovskite, this hybrid PD showed increased on-off ratio, faster response speed, and higher responsivity and detectivity. The significantly improved performance was attributed to the fact that photogenerated charges were separated efficiently, owing to the matched energy band levels between $\mathrm{ZnO}$ and perovskite. The results provide a new and promising route to integrate inorganic functional materials with perovskite for high-performance and low-cost PDs.

$\mathrm{Fu}$ et al. [97] prepared a novel $\mathrm{AgD} / \mathrm{AgCl}$ hybrid through a simple electrodeposition method using $\mathrm{AgNO}_{3}$ and CTAC and precursors. The prepared $\mathrm{AgD} / \mathrm{AgCl}$ hybrid consisted of $\mathrm{Ag}$ dendrites and $\mathrm{AgCl}$ polyhedral. Energydispersive X-ray spectroscopy (EDS) analysis and X-ray diffraction (XRD) characterizations confirmed that the hybrid synthesized through electrodeposition had high purity. The prepared $\mathrm{AgD} / \mathrm{AgCl}$ hybrid with a specific morphology exhibited an effective photosensitive property with a fast response time and good photocurrent reproducibility.

Gogurla et al. [98] demonstrated $\mathrm{ZnO}$ nanorod hybrid PDs on AuNP-embedded silk protein for flexible optoelectronics. Hybrid samples exhibited optical absorption at the band edge of $\mathrm{ZnO}$ as well as plasmonic energy due to AuNPs, making them attractive for selective UV and visible wavelength detection. The device prepared on Au-silk protein showed a much lower dark current and a higher photo to dark current ratio of $10^{5}$ compared to the control sample without AuNPs. The hybrid device also exhibited a higher specific detectivity due to higher responsivity arising from the photogenerated hole trapping by AuNPs. The proposed novel hybrid $\mathrm{ZnO}$ nanorods on silk protein can provide a pathway for new opportunities toward lowcost, biocompatible, biodegradable, flexible, and lightweight PDs.

Ouyang et al. [37] reported a novel hybrid structure of $\mathrm{BiOCl} / \mathrm{ZnO}$ film and $\mathrm{TiO}_{2} / \mathrm{ZnO}$ film by a facile spincoating method and constructed it to PDs. As shown in Figure 9, due to the formation of $\mathrm{p}$ - $\mathrm{n}$ junction between the $\mathrm{BiOCl}$ and $\mathrm{ZnO}$, compared to the obtained $\mathrm{TiO}_{2} / \mathrm{ZnO}$ hybrid film PD, the BiOCl/ZnO film PD exhibits excellent optoelectronic properties, including higher photocurrent/ responsivity and shorter response time, outperforming the pristine $\mathrm{ZnO}$ film PD counterpart. However, excess BiOCl clusters in the hybrid film would significantly block the transport path of electrons between electrodes, resulting in the decrease of photocurrent. Thus, we should put emphasis on how to develop the construction of BiOCl/ ZnO hybrid film to increase photocurrent. 

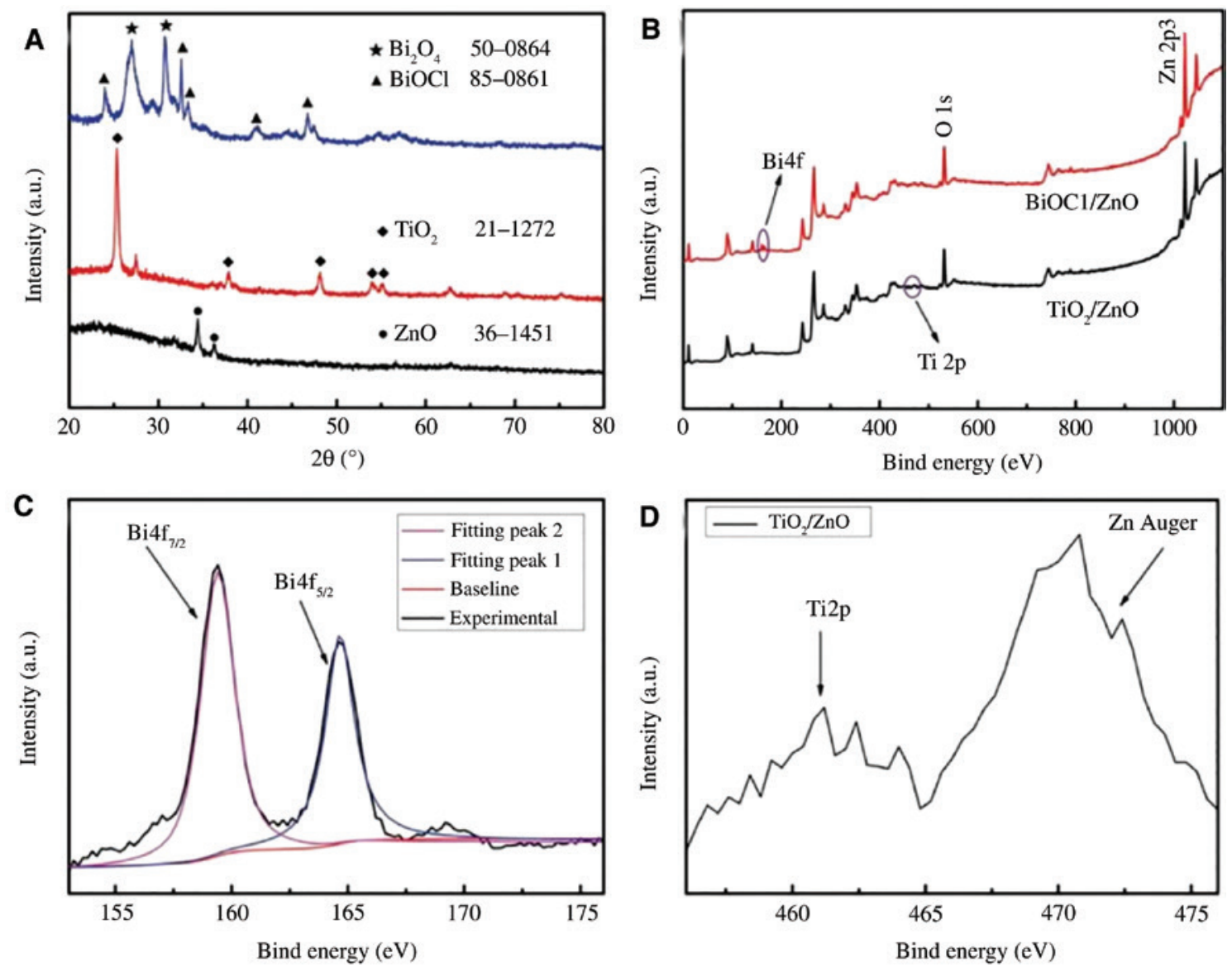

Figure 9: X-ray photoelectron spectroscopy of different films: (A) XRD patterns of the $\mathrm{ZnO}$ film (black line), $\mathrm{TiO}_{2} \mathrm{NPs}$ (red line), and $\mathrm{BiOCl}$ nanosheets (blue line). (B) Survey scan. (C) $\mathrm{Bi}_{4} \mathrm{~F}$. (D) $\mathrm{Ti}_{2} \mathrm{p}$ spectrum. Reproduced with permission from Ref. [42].

Lee et al. [99] demonstrated the fabrication of a novel hybrid PD consisting of graphene and $\mathrm{CH}_{3} \mathrm{NH}_{3} \mathrm{PbI}_{3}$ perovskite layers. The device exhibited a broad spectral photoresponsivity between 800 and $400 \mathrm{~nm}$, and the photoresponsivity and effective quantum efficiency were $180 \mathrm{~A} / \mathrm{W}$ and $5 \times 10^{4}$ at a relatively high illumination power of $1 \mu \mathrm{W}$, respectively. All of these high performances were attributed to the efficient charge transfer from the graphene to the perovskite. However, when this device showed fast rise time in the photoresponse, the relatively slow fall time hindered the performance of the PD. This problem might be solved by introducing an additional layer (on top of the device) having a lower valence band than $\mathrm{CH}_{3} \mathrm{NH}_{3} \mathrm{PbI}_{3}$. The hybrid $\mathrm{PD}$ described here is expected to contribute to the development of imaging sensors suitable for low-light photography, UV detectors with a high responsivity, and smart skin sensors combined with other functionalities.

\section{Conclusion}

This review summarizes the latest research on PDs based on low-dimensional nanomaterials. Nanostructured materials with a number of fascinating and excellent photoelectric properties have promised themselves as versatile building blocks in terms of interconnects and functional units for novel PDs. Therefore, they are expected to play an important role in improving the performance and extending the function of traditional PDs.

However, with regard to the overall perspective, the study of nanoscale PDs is still at its preliminary stage. To make full use of nanostructured materials' unique photoelectronic properties for practical applications, studies of many issues need to be urgently intensified.

In view of the basic scientific research and potential technological applications, a roadmap for further directions of nanoscale PDs is described as follows. First, the 
ability to control nanostructured materials precisely is essential for high-performance nanodevices. Second, studies focusing on improving the photocurrent of hybrid PDs is needed. Additionally, to further increase the quantum efficiency of the photodetection, a new design of nanostructures with high efficient absorption of the incident light and hot electron generation is preferred. Last but not least, the use of new materials such as lowdimensional materials will also extend the spectral range of light absorption and hot-electron generation, allowing efficiently converting the light to current signals.

Although many riddles need to be solved, behind this burgeoning field, it is no doubt that the combination of well-studied materials and established device fabrication technologies will lead to more and more exciting discoveries in the further development of PDs. In view of the complexity of researching and developing nanoscale PDs, research tools from different disciplines, including physics, chemistry, materials science, and microelectronics, need to be pulled together to solve these problems. Of course, challenges mean opportunities as well, the entirely new era of photodetection is beckoning us on, and we do hope this review will inspire an increasing number of researchers and engineers to join this game.

Acknowledgments: This work was supported by the National Nature Science Foundation of China (Grant No. 61604019) and the National Natural Science Foundation of Liaoning (Grant No. 20170540748).

\section{References}

[1] Chen H, Liu K, Hu L, Al-Ghamdi A, Fang X. New concept ultraviolet photodetectors. Mater. Today 2015, 18, 493-502.

[2] Ren X, Li Z, Huang Z, Sang D, Qiao H, Qi X, LI J, Zhong J, Zhang H. Environmentally robust black phosphorus nanosheets in solution: application for self-powered photodetector. Adv. Funct. Mater. 2017, 27, 1606834.

[3] Eswaraiah V, Zeng Q, Long Y, Liu Z. Black phosphorus nanosheets: synthesis, characterization and applications. Small 2016, 12, 3480-3502.

[4] Ouyang W, Teng F, Fang X. High performance BiOCI nanosheets/ $\mathrm{TiO}_{2}$ nanotube arrays heterojunction UV photodetector: the influences of self-induced inner electric fields in the $\mathrm{BiOCl}$ nanosheets. Adv. Funct. Mater. 2018, 28, 1707178.

[5] Wang F, Hu X, Niu X, Xie J, Chu S, Gong Q. Low-dimensional materials-based field-effect transistors. J. Mater. Chem. C 2018, 6, 924-941.

[6] Zheng S, Wang D, Tian Y, Jiang L. Hierarchical MoS, nanosheet@ $\mathrm{TiO}_{2}$ nanotube array composites with enhanced photocatalytic and photocurrent performances. Small 12, 1527-1536.
[7] Xiang J, Liu Z. Photodetectors based on sensitized twodimensional transition metal dichalcogenides - a review. J. Mater. Res. 2017, 32, 4115-4131.

[8] Hu K, Chen H, Jiang M, Teng F, Zheng L, Fang X. Broadband photoresponse enhancement of a high performance t-Se microtube photodetector by plasmonic metallic nanoparticles. Adv. Funct. Mater. 2016, 26, 6641-6648.

[9] Bao Y, Fang Z. Plasmon-enhanced photodetection in nanostructures. Nanotechnol. Rev. 2015, 4, 325-336.

[10] Pyo S, Kim W, Jung, Jung H, Choi J, Kim J. Heterogeneous integration of carbon-nanotube-graphene for high-performance, flexible, and transparent photodetectors. Small 2017, 13, 1700918.

[11] Wang T, Hu S, Chamlagain B, Hong T, Zhou Z, Weiss S, Xu Y. Visualizing light scattering in silicon waveguides with black phosphorus photodetectors. Adv. Mater. 2016, 28, $7162-7166$.

[12] Kang D, Jeon M, Jang S, Choi W, Kim K, Kim J, Lee S, Yeom G, Park J. Self-assembled layer (SAL)-based doping on black phosphorus (BP) transistor and photodetector. ACS Photonics 2017, 4, 1822-1830.

[13] Schuler S, Schall D, Neumaier D, Dobusch L, Bethge O, Schwarz $B$, Krall M, Mueller T. Controlled generation of a pn-junction in a waveguide integrated graphene photodetector. Nano Lett. 2016, 16, 7107-7112.

[14] Kang P, Wang M, Knapp P, Nam S. Crumpled graphene photodetector with enhanced, strain-tunable, and wavelength-selective photoresponsivity. Adv. Mater. 2016, 28, 4639-4645.

[15] Fang J, Wang D, DeVault C, Chung T, Chen Y, Boltasseva A, Shalaev V, Kildishev A. Enhanced graphene photodetector with fractal metasurface. Nano Lett. 2017, 17, 57-62.

[16] Xia F, Wang H, Xiao D, Dubey M, Ramasubramania A. Two-dimensional material nanophotonics. Nat. Photonics 2014, 8, 899.

[17] Radisavljevic B, Radenovic A, Brivio J, Giacometti V, Kis A. Single-layer MoS 2 transistors. Nat. Nanotechnol. 2011, 6, 147.

[18] Wang Q, Zadeh K, Kis A, Coleman J, Strano M. Electronics and optoelectronics of two-dimensional transition metal dichalcogenides. Nat. Nanotechnol. 2012, 7, 699.

[19] Mak K, Lee C, Hone J, Shan J, Heinz T. Atomically thin MoS: a new direct-gap semiconductor. Phys. Rev. Lett. 2010, 105, 136805.

[20] Ye L, Wang P, Luo W, Gong F, Liao L, Liu T, Tong L, Zang J, Xu J, $\mathrm{Hu}, \mathrm{W}$. Highly polarization sensitive infrared photodetector based on black phosphorus-on-WSe ${ }_{2}$ photogate vertical heterostructure. Nano Energy 2017, 37, 53-60.

[21] Gomathi P, Sahatiya P, Badhulika S. Large-area, flexible broadband photodetector based on ZnS-MoS 2 hybrid on paper substrate. Adv. Funct. Mater. 2017, 27, 1701611.

[22] Li L, Yu Y, Ye G, Ge Q, Ou X, Wu H, Feng D, Chen X, Zhang Y. Black phosphorus field-effect transistors. Nat. Nanotechnol. 2014, 9, 372.

[23] Bridgman P. Two new modification of phosphorus. J. Am. Chem. Soc. 1914, 36, 1344-1363.

[24] Xiong X, Li X, Huang M, Li T, Gao T, Wu Y. High performance black phosphorus electronic and photonic devices with HfLaO dielectric. IEEE Electron. Device Lett. 2018, 39, 127-130.

[25] Miao J, Song B, Xu Z, Cai L, Zhang S, Dong L, Wang C. Single pixel black phosphorus photodetector for NIR imaging. Small 2018, 14, 1702082. 
[26] Youngblood N, Chen C, Koester S, Li M. Waveguide-integrated black phosphorus photodetector with high responsivity and low dark current. Nat. Photonics 2015, 9, 247.

[27] Deng Y, Luo Z, Conrad N, Liu H, Gong Y, Najmaei S, Ajayan P, Lou J, Xu X, Ye P. Black phosphorus-monolayer $\mathrm{MoS}_{2}$ van der Waals heterojunction p-n diode. ACS Nano 2014, 8, 8292-8299.

[28] Miao J, Xu Z, Li Q, Bowman A, Zhang S, Hu W, Zhou Z, Wang C. Vertically stacked and self-encapsulated van der Waals heterojunction diodes using two-dimensional layered semiconductors. ACS Nano 2017, 11, 10472-10479.

[29] Li H, Ye L, Xu J. High-performance broadband floating-base bipolar phototransistor based on $\mathrm{WSe} / \mathrm{BP} / \mathrm{MoS}_{2}$ heterostructure. ACS Photonics 2017, 4, 823-829.

[30] Ding Y, Shi J, Xia C, Zhang M, Du J, Huang P, Wu M, Wang H, Cen Y, Pan S. Enhancement of hole mobility in InSe monolayer via an InSe and black phosphorus heterostructure. Nanoscale 2017, 9, 14682-14689.

[31] Ye L, Li H, Chen Z, Xu J. NIR photodetector based on $\mathrm{MoS}_{2}$ /black phosphorus heterojunction. ACS Photonics 2016, 3, 692-699.

[32] Wu J, Koon G, Xiang D, Han C, Toh C, Kulkarni E, Verzhbitskiy I, Carvalho A, Koenig S, Eda G, Chen W, Neto A, Ozyilmaz B. Colossal ultraviolet photoresponsivity of few-layer black phosphorus. ACS Nano 2015, 9, 8070-8077.

[33] Lim S, Hong S, Hwang S, Choi W, Kim S, Park H, Jeong M. Synthesis of Al-doped $\mathrm{ZnO}$ nanorods via microemulsion method and their application as a CO Gas sensor. J. Mater. Sci. Technol. 2015, 31, 639-644.

[34] Teng F, Zheng L, Hu K, Chen H, Li Y, Zhang Z, Fang X. Surface oxide thin layer of copper nanowires enhanced UV selective response of ZnO film photodetector. J. Mater. Chem. C 2016, 4, 8416-8421.

[35] Fang X, Bando Y, Liao M, Gautam U, Zhi C, Dierre B, Liu B, Zhai T, Sekiguchi T, Koide Y, Golberg D. Single-crystalline ZnS nanobelts as ultraviolet-light sensors. Adv. Mater. 2009, 21, 2034-2039.

[36] Zhao B, Wang F, Chen H, Zheng L, Su L, Zhao D, Fang X. An ultrahigh responsivity $\left(9.7 \mathrm{~mA} \mathrm{~W}^{-1}\right)$ self-powered solar-blind photodetector based on individual $\mathrm{ZnO}-\mathrm{Ga}_{2} \mathrm{O}_{3}$ heterostructures. Adv. Funct. Mater. 2017, 27, 1700264.

[37] Ouyang W, Teng F, Jiang M, Fang X. ZnO film UV photodetector with enhanced performance: heterojunction with $\mathrm{CdMoO}_{4}$ microplates and the hot electron injection effect of Au nanoparticles. Small 2017, 13, 1702177.

[38] Chen H, Liu K, Chen X, Zhang Z, Fan M, Jiang M, Xie X, Zhao H, Shen D. Realization of a self-powered ZnO MSM UV photodetector with high responsivity using an asymmetric pair of Au electrodes. J. Mater. Chem. C 2014, 2, 9689-9694.

[39] Hu K, Teng F, Zheng L, Yu P, Zhang Z, Chen H, Fang X. Binary response $\mathrm{Se} / \mathrm{ZnO} \mathrm{p}$-n heterojunction UV photodetector with high on/off ratio and fast speed. Laser Photonics Rev. 2017, 11, 1600257.

[40] Gedamu D, Paulowicz I, Kaps S, Lupan O, Wille S, Haidarschin G, Mishra Y, Adelung R. Rapid fabrication technique for interpenetrated ZnO nanotetrapod networks for fast UV sensors. Adv. Mater. 2014, 26, 1541-1550.

[41] Chen H, Liu H, Zhang Z, Hu K, Fang X. Nanostructured photodetectors: from ultraviolet to terahertz. Adv. Mater. 2016, 28, 403-433.
[42] Teng F, Ouyang W, Li Y, Zheng L, Fang X. Novel structure for high performance UV photodetector based on $\mathrm{BiOCl} / \mathrm{ZnO}$ hybrid film. Small 2017, 13, 1700156.

[43] Ning Y, Zhang Z, Feng T, Fang X. Novel transparent and selfpowered UV photodetector based on crossed $\mathrm{ZnO}$ nanofiber array homojunction. Small 2018, 14, 1703754.

[44] Zhao B, Wang F, Chen H, Wang Y, Jiang M, Fang X, Zhao D. Solar-blind avalanche photodetector based on single $\mathrm{ZnO}$ $\mathrm{Ga}_{2} \mathrm{O}_{3}$ core-shell microwire. Nano Lett. 2015, 15, 3988-3993.

[45] Duan L, Wei F, Fan J, Yu X, Zhang W, Zhang Y, He F, Cheng X, Tian Y. Low-cost fabrication of UV photodetector based on hexagonal nanocrystal ZnO: Al/p-Si heterojunction. Nano 2016, 11, 1650019.

[46] Chen H, Yu P, Zhang Z, Teng F, Zheng L, Hu K, Fang X. Ultrasensitive self-powered solar-blind deep-ultraviolet photodetector based on all-solid-state polyaniline/MgZnO bilayer. Small 2016, 12, 5809-5816.

[47] Xu J, Yang W, Chen H, Zheng L, Hu M, Li Y, Fang X. Efficiency enhancement of $\mathrm{TiO}_{2}$ self-powered UV photodetectors using transparent Ag nanowires electrode. J. Mater. Chem. C 2018, 6, 3334-3340.

[48] Su L, Yang W, Cai J, Chen H, Fang X. Self-powered ultraviolet photodetectors driven by built-in electric field. Small 2017, 13, 1701687.

[49] Yu P, Hu K, Chen H, Zheng L, Fang X. Novel p-p heterojunctions self-powered broadband photodetectors with ultrafast speed and high responsivity. Adv. Funct. Mater. 2017, 27, 1703166.

[50] Purusothaman Y, Alluri N, Chandrasekhar A, Vivekanantha V, Kim S. Regulation of charge carrier dynamics in $\mathrm{ZnO}$ microarchitecture-based UV/visible photodetector via photonicstrain induced effects. Small 2018, 14, 1703044.

[51] Chen M, Zhao B, Hu G, Fang X, Wang H, Wang L, Luo J, Han X, Wang $X$, Pan C, Wang Z. Piezo-phototronic effect modulated deep UV photodetector based on $\mathrm{ZnO}-\mathrm{Ga}_{2} \mathrm{O}_{3}$ heterojunction microwire. Adv. Funct. Mater. 2018, 28, 1706379.

[52] Zheng L, Hu K, Teng F, Fang X. Novel UV-visible photodetector in photovoltaic mode with fast response and ultrahigh photosensitivity employing $\mathrm{Se} / \mathrm{TiO}_{2}$ nanotubes heterojunction. Small 2017, 13, 1602448.

[53] Teng F, Hu K, Ouyang W, Fang X. Photoelectric detectors based on inorganic p-type semiconductor materials. Adv. Mater. 2018, 30, 1706262.

[54] Lijima S, Ichihashi T. Single-shell carbon nanotubes of I-nm diameter. Nature 1993, 363, 603.

[55] Liang S, Ma Z, Wu G, Wei N, Huang L, Huang H, Liu H, Wang $\mathrm{S}$, Peng L. Microcavity-integrated carbon nanotube photodetectors. ACS Nano 2016, 10, 6963-6971.

[56] Pradhan B, Setyowati K, Liu H, Waldeck D, Chen J. Carbon nanotube-polymer nanocomposite infrared sensor. Nano Lett. 2008, 8, 1142-1146.

[57] Hecht D, Ramirez R, Briman M, Artukovic E, Chichak K, Stoddart J, Gruner G. Bioinspired detection of light using a porphyrin-sensitized single-wall nanotube field effect transistor. Nano Lett. 2006, 6, 2031-2036.

[58] Rogalski A, Antoszewski J, Faraone L. Third-generation infrared photodetector arrays. J. Appl. Phys. 2009, 105, 4.

[59] Weisman R, Bachilo S. Dependence of optical transition energies on structure for single-walled carbon nanotubes in aqueous suspension: an empirical Kataura plot. Nano Lett. 2003, 3, $1235-1238$ 
[60] Freitag M, Low T, Zhu W, Yan H, Xia F, Avouris P. Photocurrent in graphene harnessed by tunable intrinsic plasmons. Nat. Commun. 2013, 4, 1951.

[61] Sobhani A, Knight M, Wang Y, Zheng B, King N, Brown L, Fang Z, Nordlander P, Halas N. Narrowband photodetection in the near-infrared with a plasmon-induced hot electron device. Nat. Commun. 2013, 4, 1643.

[62] Pyo S, Kim W, Jung H, Choi J, Kim J. Heterogeneous integration of carbon-nanotube- graphene for high-performance, flexible, and transparent photodetectors. Small 2017, 13, 1700918.

[63] Huang H, Wang F, Liu Y, Wang S, Peng L. Plasmonic enhanced performance of infrared detector based on carbon nanotube films. ACS Appl. Mater. Interface 2017, 9, 12743-12749.

[64] Zhang L, Yang T, Shen L, Fang Y, Dang L, Zhou N, Guo X, Hong Z, Yang Y, Wu H, Huang J, Liang Y. Toward highly sensitive polymer photodetectors by molecular engineering. Adv. Mater. 2015, 27, 6496-6503.

[65] Wen Y, Wang Q, Yin L, Liu Q, Wang F, Wang F, Wang Z, Liu K, Xu K, Huang Y, Shifa T, Jiang C, Xiong J, He J. Epitaxial 2D PbS nanoplates arrays with highly efficient infrared response. $A d v$. Mater. 2016, 28, 8051-8057.

[66] Saran R, Curry R. Lead sulphide nanocrystal photodetector technologies. Nat. Photonics 2016, 10, 81.

[67] Wu W, Chakrabortty S, Guchhait A, Wong G, Dalapati G, Lin M, Chan Y. Solution-processed 2D PbS nanoplates with residual $\mathrm{Cu}_{2} \mathrm{~S}$ exhibiting low resistivity and high infrared responsivity. Chem. Mater. 2016, 28, 9132-9138.

[68] Schliehe C, Juarez B, Pelletier M, Jander S, Greshnykh D, Nagel M, Meyer A, Foerster S, Kornowski A, Weller H. Ultrathin PbS sheets by two-dimensional oriented attachment. Science 2010, 329, 550-553.

[69] Zhang H, Savitzky B, Yang J, Newman J, Perez K, Hyun B, Kourkoutis L, Hanrath T, Wise F. Colloidal synthesis of PbS and PbS/ CdS nanosheets using acetate-free precursors. Chem. Mater. 2015, 28, 127-134.

[70] Li H, Zhitomirsky D, Grossman J. Tunable and energetically robust $\mathrm{PbS}$ nanoplatelets for optoelectronic applications. Chem. Mater. 2016, 28, 1888-1896.

[71] Shen L, Zhang Y, Bai Y, Zheng X, Wang Q, Huang J. A filterless, visible-blind, narrow-band, and NIR photodetector with a gain. Nanoscale 2016, 8, 12990-12997.

[72] Lee J, Kim D, Baek S, Yu H, So F. Inorganic UV-visible-SWIR broadband photodetector based on monodisperse $\mathrm{PbS}$ nanocrystals. Small 2016, 12, 1328-1333.

[73] Qiao K, Deng H, Yang X, Dong D, Li M, Hu L, Liu H, Song H, Tang J. Spectra-selective PbS QD infrared photodetectors. Nanoscale 2016, 8, 7137-7143.

[74] Mishra N, Mukherjee B, Xing G, Chakrabortty S, Guchhait $\mathrm{A}$, Lim J. Cation exchange synthesis of uniform $\mathrm{PbSe} / \mathrm{PbS}$ core/shell tetra-pods and their use as NIR photodetectors. Nanoscale 8, 14203-14212.

[75] Lee J, Kim D, So F. Unraveling the gain mechanism in high performance solution-processed PbS infrared PIN photodiodes. Adv. Funct. Mater. 2015, 25, 1233-1238.

[76] Li X, Yu D, Chen J, Wang Y, Cao F, Wei Y, Wu Y, Wang L, Zhu Y, Sun Z, Ji J, Shen Y, Sun H, Zeng H. Constructing fast carrier tracks into flexible perovskite photodetectors to greatly improve responsivity. ACS Nano 2017, 11, 2015-2023.
[77] Li X, Yu D, Cao F, Gu Y, Wei Y, Wu Y, Song J, Zeng H. Healing all-inorganic perovskite films via recyclable dissolutionrecyrstallization for compact and smooth carrier channels of optoelectronic devices with high stability. Adv. Funct. Mater. 2016, 26, 5903-5912.

[78] Waleed A, Tavakoli M, Gu L, Hussain S, Zhang D, Poddar S, Wang Z, Zhang R, Fan Z. All inorganic cesium lead iodide perovskite nanowires with stabilized cubic phase at room temperature and nanowire array-based photodetectors. Nano Lett. 2017, 17, 4951-4957.

[79] Liu C, Wang K, Du P, Wang E, Gong X, Heeger A. Ultrasensitive solution-processed broad-band photodetectors using $\mathrm{CH}_{3} \mathrm{NH}_{3} \mathrm{Pbl}_{3}$ perovskite hybrids and $\mathrm{PbS}$ quantum dots as light harvesters. Nanoscale 2015, 7, 16460-16469.

[80] Ren Z, Sun J, Li H, Mao P, Wei Y, Zhong X, Hu J, Yang S, Wang J. Bilayer $\mathrm{PbS}$ quantum dots for high-performance photodetectors. Adv. Mater. 2017, 29, 1702055.

[81] Wen Y, Yin L, He P, Wang Z, Zhang X, Wang Q, Shifa T, Xu K, Wang F, Zhan X, Wang F, Jiang C, He J. Integrated high-performance infrared phototransistor arrays composed of nonlayered $\mathrm{PbS}-\mathrm{MoS}_{2}$ heterostructures with edge contacts. Nano Lett. 2016, 16, 6437-6444.

[82] Liu C, Peng H, Wang K, Wei C, Wang Z, Gong X. PbS quantum dots-induced trap-assisted charge injection in perovskite photodetectors. Nano Energy 2016, 30, 27-35.

[83] Wei Y, Ren Z, Zhang A, Mao P, Li H, Zhong X, Li W, Yang S, Wang J. Hybrid organic/PbS QD bilayer photodetector with low dark current and high detectivity. Adv. Funct. Mater. 2018, 28, 1706690.

[84] Liu Z, Luo T, Liang B, Chen G, Yu G, Xie X, Chen D, Shen G. High-detectivity InAs nanowire photodetectors with spectral response from ultraviolet to NIR. Nano. Res. 2013, 6, 775-783.

[85] Deng K, Li L. CdS nanoscale photodetectors. Adv. Mater. 2014, 26, 2619-2635.

[86] Xiong X, Zhang Q, Gan L, Zhou X, Xing X, Li H, Zhai T. Geometry dependent photoconductivity of $\operatorname{In}_{2} S_{3}$ kinks synthesized by kinetically controlled thermal deposition. Nano. Res. 2016, 9, 3848-3857.

[87] Li L, Fang X, Zhai T, Liao M, Gautam U, Wu X, Koide Y, Bando Y, Golberg D. Electrical transport and high-performance photoconductivity in individual $\mathrm{ZrS}_{2}$ nanobelts. Adv. Mater. 2010, 22, 4151-4156.

[88] Cao F, Tian W, Gu B, Ma Y, Lu H, Li L. High-performance UV-vis photodetectors based on electrospun $\mathrm{ZnO}$ nanofiber-solution processed perovskite hybrid structures. Nano. Res. 2017, 10, 2244-2256.

[89] Zhao Y, Zhu K. Organic-inorganic hybrid lead halide perovskites for optoelectronic and electronic applications. Chem. Soc. Rev. 2016, 45, 655-689.

[90] Dou L, Yang Y, You J, Hong Z, Chang W, Li G, Yang Y. Solutionprocessed hybrid perovskite photodetectors with high detectivity. Nat. Commun. 2014, 5, 5404.

[91] Sutherland B, Jognston A, Ip A, Xu J, Adinolfi V, Kanjanaboos P, Sargent $E$. Sensitive, fast, and stable perovskite photodetectors exploiting interface engineering. ACS Photonics 2015, 2 , 1117-1123.

[92] Chen J, Zhou S, Jin S, Li H, Zhai T. Crystal organometal halide perovskites with promising optoelectronic applications. J. Mater. Chem. C 2016, 4, 11-27. 
[93] Zhao Y, Yang S, Zhao J, Zou B. PbS quantum dots based organic-inorganic hybrid infrared detecting and display devices. Mater. Lett. 2017, 196, 176-178.

[94] Liu Z, Parvez K, Li R, Dong R, Feng X, Müllen K. Transparent conductive electrodes from graphene/PEDOT: PSS hybrid inks for ultrathin organic photodetectors. Adv. Mater. 2015, 27, 669-675.

[95] Kim M, Kang P, Leem J, Nam S. Stretchable crumpled graphene photodetector with plasmonically enhanced photoresponsivity. Nanoscale 2017, 9, 4058-4065.

[96] Ni Z, Ma L, Du S, Xu Y, Yuan M, Fang H, Wang Z, Xu M, Li D, Yang J, Hu W, Pi X, Yang D. Plasmonic silicon quantum dots enabled high-sensitivity ultrabroadband photodetection of graphenebased hybrid phototransistors. ACS Nano 2017, 11, 9854-9862.

[97] Fu L, Wang A, Zheng Y, Cai W, Fu Z. Electrodeposition of Ag dendrites/AgCl hybrid film as a novel photodetector. Mater. Lett. 2015, 142, 119-121.

[98] Gogurla N, Kundu S, Ray S. Gold nanoparticle-embedded silk protein-ZnO nanorod hybrids for flexible bio-photonic devices. Nanotechnology 2017, 28, 145202.

[99] Lee Y, Kwon J, Hwang E, Ra C, Yoo W, Ahn J, Park J, Cho J. Highperformance perovskite-graphene hybrid photodetector. Adv. Mater. 2014, 27, 1402271.

\section{Bionotes}

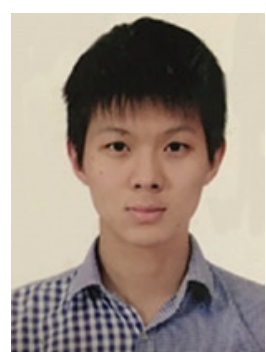

\section{Zhenhui Li}

School of Information and Control

Engineering, Shenyang Jianzhu University, Shenyang, China

Zhenhui Li studies at Shenyang Jianzhu University as an undergraduate and majors in electrical and intelligent building. His main research is electron devices and hopes to contribute to the development of PDs.

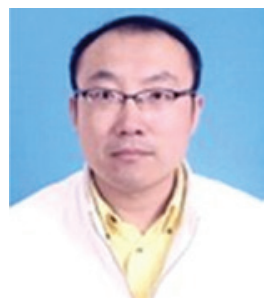

Ke Xu

School of Information and Control

Engineering, Shenyang Jianzhu University, Shenyang, China,

xksky1234@163.com

Ke Xu PhD is a professor at Shenyang Jianzhu University. He received his master's degree in control theory and control engineering in March 2005 from Shenyang Jianzhu University. In March 2008, he joined the State Key Laboratory at the Shenyang Institute of Automation (SIA), CAS. He received his $\mathrm{PhD}$ in pattern recognition and intelligent system in July 2012. He finished his postdoctoral research in mechanical and electrical engineering in January 2016 at the SIA, CAS.

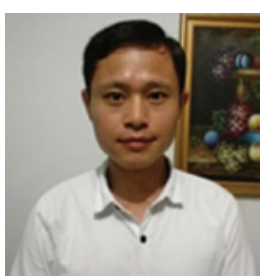

Fanan Wei

School of Mechanical Engineering and Automation, Fuzhou University, Fuzhou, China

Fanan Wei received his undergraduate degree in automation in June 2009 from Huazhong University of Science and Technology (Wuhan, China). In September 2009, he joined the State Key Laboratory at the SIA, CAS, and received his PhD in mechanical and electrical engineering in December 2015. Then, he joined the faculty at the School of Mechanical Engineering and Automation in Fuzhou University (Fujian, China). 